\title{
Tracer Conservation for Three-Dimensional, Finite-Element, Free-Surface, Ocean Modeling on Moving Prismatic Meshes
}

\author{
LAURENT WHITE \\ Centre for Systems Engineering and Applied Mechanics, and G. Lemaître Institute of Astronomy and Geophysics, \\ Université Catholique de Louvain, Louvain-la-Neuve, Belgium \\ VINCENT LEGAT \\ Centre for Systems Engineering and Applied Mechanics, Université Catholique de Louvain, Louvain-la-Neuve, Belgium \\ ERIC DELEERSNIJDER \\ G. Lemaître Institute of Astronomy and Geophysics, and Centre for Systems Engineering and Applied Mechanics, \\ Université Catholique de Louvain, Louvain-la-Neuve, Belgium
}

(Manuscript received 10 January 2007, in final form 10 April 2007)

\begin{abstract}
Large-scale free-surface ocean models designed to run over climatic time scales are required to globally conserve the volume and any tracer up to machine precision. In addition, local consistency is critical and requires that the discrete tracer equation preserve constants in a closed domain and if there is no tracer source or sink. Local consistency, together with monotonicity, will ensure that no spurious tracer extrema occur. A three-dimensional, finite-element, shallow-water model is presented. The mesh is unstructured in the horizontal, extruded in the third dimension, and made up of multiple layers of prisms. In addition, the mesh is allowed to move in the vertical and adapts itself to the free-surface motions. It is shown that achieving consistency requires a discrete compatibility between the tracer and continuity equations. In addition, to ensure global tracer conservation in a consistent way, a discrete compatibility between the tracer, continuity, and free-surface elevation equations must be fulfilled. It is suggested that this compatibility constraint, together with the use of a numerically stable scheme, severely restricts the choice of usable finite-element spatial discretizations. A consistent and conservative time-stepping algorithm is described for which a unique time step is used. It is suggested that future research is needed in order to design a consistent and conservative split-explicit algorithm. Some illustrative test cases are presented in which the method is shown to satisfy all conservation properties. A few experiments in which consistency breaks down are carried out, and the consequences of this breakdown process are investigated.
\end{abstract}

\section{Introduction}

The intrinsic flexibility of unstructured meshes is compelling for numerical ocean modeling. Complex topographic features, such as coastlines, islands, narrow straits, and sills, can faithfully be represented by locally increasing the mesh resolution and because there is no constraint on the mesh topology (e.g., Legrand et al. 2006). The resolution can also be altered based upon

Corresponding author address: Laurent White, Princeton University/GFDL, 201 Forrestal Road, Princeton, NJ 08542.

E-mail: laurentw@princeton.edu other criteria such as that based on the bathymetry (Gorman et al. 2006; Legrand et al. 2007) or a model variable (Legrand et al. 2000). In the latter case, the mesh can also be dynamically adapted in the course of the simulation (Piggott et al. 2005; Power et al. 2006). Over the last decade, motivated by these concepts, increasing efforts have been directed toward the development of ocean models based on unstructured meshes. Three classes of numerical methods can readily handle unstructured meshes: the finite-volume (FV), the spectral-element (SE), and the finite-element (FE) methods.

To a certain extent, each of these methods has been gaining popularity in the ocean modeling community.

DOI: 10.1175/2007MWR2137.1 
For example, the finite-volume method is employed by Casulli and Walters (2000), Ham et al. (2005), and Fringer et al. (2006). The SE method is used by Iskandarani et al. (1995, 2003). The use of the FE method for coastal, shelf, and estuarine areas started somewhat earlier Walters and Werner 1989; Lynch and Werner 1987, 1991). During the 1990s, aside from some results obtained with diagnostic finite-element ocean models (Myers and Weaver 1995; Greenberg et al. 1998), we did not really see any revolutionary change. Over the last $5 \mathrm{yr}$, with new projects involving finite-element global ocean models, such as the Finite-Element Ocean Circulation Model (FEOM) from the Alfred Wegener Institute for Polar and Marine Research, in Bremerhaven, Germany; the Imperial College Ocean Model (ICOM) from Imperial College, in London, United Kingdom; and the Second-Generation Louvain-laNeuve Ice-Ocean Model (SLIM) from the Université Catholique de Louvain, in Louvain-la-Neuve, Belgium, we have been witnessing a new wave of thriving development (and funding) of prognostic finite-element ocean models. As warranted by the literature, the FE method appears to be the most promising (e.g., the recent special issues of Ocean Modelling, Vol. 10, Nos. 1-2, and Vol. 15, Nos. 1-2). There might be two main reasons for this. While all of the methods mentioned can handle unstructured meshes, the finite-element method offers additional flexibility in the choice of interpolation (it can be of low or high order and be continuous or discontinuous) and is sustained by a rigorous mathematical framework, in which a priori and a posteriori error estimates can be established.

The first developments of finite-element marine models were based on the wave continuity equation (Lynch and Gray 1979), whereby the primitive shallowwater equations are manipulated to form a wave equation for the free-surface elevation. This formulation does not suffer from spurious oscillations occurring when using the primitive equations and the same interpolation for the velocity and the elevation. The generalization of the original method led to the generalized wave continuity equation (GWCE), documented by Kinnmark (1986) and Kolar et al. (1994). The GWCE has been extensively used over the past $20 \mathrm{yr}$ with successful applications in coastal regions for tidal predictions (Walters 1992; Lynch and Naimie 1993; Lynch et al. 1996; Fortunato et al. 1997; Cushman-Roisin and Naimie 2002). Despite these encouraging applications and the ongoing research involved in improving the method, it remains plagued by two caveats. GWCEbased models are subject to advective instabilities (Kolar et al. 1994) and the GWCE form sacrifices the primitive continuity equation; thus, the primitive form is no longer satisfied in a discrete sense, which implies continuity (or mass) imbalances (Dawson et al. 2006; Massey and Blain 2006), rendering the method less suitable for coupling with transport equations, let alone for long time integrations (more than several years) in which conservation is crucial.

Since the end of the 1990s, more effort has been directed toward finding a mixed finite-element pair for the primitive shallow-water equations that does not support spurious oscillations (Le Roux et al. 1998). Aware of the limitations of the GWCE and motivated by the desire to develop primitive equation finiteelement ocean models, research toward this goal intensified (Le Roux 2001, 2005; Hanert et al. 2003; Walters 2006; White et al. 2006b; Le Roux et al. 2005, 2007). Concerns over some of the early issues of the method that were often cited as reasons not to use it-such as spurious oscillations, unphysical wave scattering due to the unstructured character of the mesh, and lack of mass conservation-are starting to wither. Nowadays, applications of FE shallow-water models based on the primitive equations are becoming much less of an exception (Nechaev et al. 2003; Danilov et al. 2004, 2005; Ford et al. 2004a,b; Pain et al. 2005; Hanert et al. 2005; Labeur and Pietrzak 2005; Walters 2006; White et al. 2006a; White and Deleersnijder 2007; White 2007) and this trend is unlikely to lose its momentum.

Finite-element methods based on the primitive equations encompass both the continuous Galerkin (CG) and discontinuous Galerkin (DG) methods. Application of the latter to the shallow-water equations is newer than of the former and has much to offer for solving hyperbolic systems of equation (Flaherty et al. 2002) and advection-dominated flows in general (e.g., Hanert et al. 2004; Kubatko et al. 2006b). Furthermore, the method is known to conserve flow properties elementwise in the intuitive meaning of the FV method [as opposed to the local conservation property of CG methods described by Hughes et al. (2000)]. At least for now, DG methods remain less mature than CG methods for ocean modeling. They have nonetheless been gaining popularity for modeling shallow-water flows as well (Schwanenberg et al. 2000; Aizinger and Dawson 2002; Schwanenberg and Harms 2004; Nair et al. 2005; Kubatko et al. 2006b; White et al. 2006b; Bernard et al. 2007) and are particularly well suited for transport problems (Kubatko et al. 2006a).

Despite these recent achievements, many challenges still lie ahead of us. Admittedly, switching from structured meshes to unstructured meshes requires developers to build new models from scratch rather than con- 
verting existing ones piece by piece. Quite ironically, with this approach, we find ourselves in need of addressing issues that have been solved in finitedifference (FD) models but that remain somewhat problematic or controversial with finite-element models. Ensuring global tracer conservation while preserving consistency (i.e., compatibility) between equations in finite-element shallow-water models is one of these issues and is at the core of this paper. This very issue has already been thoroughly addressed in FD models (Deleersnijder 1993; Roullet and Madec 2000; Griffies et al. 2001; Gross et al. 2002; Campin et al. 2004; Griffies 2004). A common mistaken criticism of the CG method is the lack of elementwise conservation. Several attempts to prove this criticism wrong have been made by defining interelement fluxes appropriately using so-called nodal fluxes (Hughes et al. 2000; Berger and Howington 2002). Those efforts, however, have not really mitigated the widespread belief that the finiteelement method is not conservative and, thereby, not suitable for ocean modeling. In this paper, we demonstrate how to achieve global tracer conservation and consistency in a three-dimensional, free-surface, FE shallow-water model on moving meshes. The model, whose description and validation are presented by White (2007), solves the hydrostatic primitive equations and conserves tracers in a consistent way. Global tracer conservation is attained if the volume integral of any tracer concentration within the domain is fixed in time, in the absence of boundary fluxes and source terms. It is unquestionable to us that any ocean model aimed at running over climatic time scales must achieve global conservation. Consistency or compatibility is meant in the following sense: in a discrete model, set a tracer concentration to a uniform value throughout the domain and let the free surface undulate, yet do not add boundary fluxes; the tracer concentration must maintain the same uniform value at all times. If this property is not satisfied, artificial tracer extrema might appear with the indirect effect of generating unphysical currents (in case the tracer is temperature or salinity). For this reason, it is indispensable that any ocean model should solve the elevation, continuity, and tracer equations in a consistent way. That is, they must be discretely compatible. Note that in FD models, consistency is commonly referred to as local conservation (Griffies et al. 2001; Campin et al. 2004; Griffies 2004). This is so because ensuring conservation at the cell level in terms of fluxes implies consistency. This is not necessarily true in FE models where the scheme could be locally conservative in terms of nodal fluxes without solving the equations consistently. In addition to these properties, we opted for a free-surface formulation.
Rigid-lid formulations are now deemed obsolete for several reasons (Killworth et al. 1991; Deleersnijder and Campin 1995; Griffies et al. 2000); one of them being the inability to easily account for surface freshwater fluxes. Finally, the domain is time dependent to accommodate free-surface motions. All layers within the domain are free to move in the vertical so that the free-surface displacement is distributed over the vertical to avoid the occurrence of overly thin layers near the surface. As will be shown, the volume change due to freshwater input (output) automatically leads to the dilution (concentration) of salt without having to resort to salt fluxes.

In this paper, we derive sufficient conditions regarding the FE spatial discretization to ensure global tracer conservation and consistency. We show that the same interpolation in the horizontal must be used for the elevation, the vertical velocity, and the tracers. Moreover, the same interpolation in the vertical must be used for the vertical velocity and the tracers. It is also demonstrated that computing the vertical velocity via the continuity equation, integrating it upward or downward and subject to one boundary condition, is well posed and does not lead to any accumulation of errors as was earlier suggested (Lynch and Naimie 1993; Muccino et al. 1997; Danilov et al. 2004). This remains true as long as the upper boundary of the domain coincides with the free surface and a consistent horizontal velocity is used. We also describe a time-stepping algorithm that satisfies all compatibility constraints and, so, ensures conservation and consistency. The algorithm works in both cases of nonlinear and linearized freesurface elevation equations. When mode splitting is opted for, the horizontal velocity must be corrected prior to computing the vertical velocity in order to ensure a discrete compatibility between the elevation and continuity equations. Some illustrative test cases are presented where the method is shown to satisfy all conservation properties. We also carry out a few experiments where consistency breaks down and investigate the consequences therefrom. We hope that this paper will successfully address some of the misunderstandings between traditional ocean modelers and those scientists willing to apply finite-element methods to geophysical flow problems.

\section{Mathematical formulation}

We shall now describe the minimum set of equations needed in our study. In this section, all classical conservation properties are inferred from the continuous equations. In section 3, we will derive a set of conditions for which these properties carry over to the dis- 
crete equations. Let $\Omega(t)$ be the three-dimensional, time-dependent domain of interest. It is bounded below by the seabed, defined by $\Gamma_{b}$, and above by the free surface, defined by $\Gamma_{s}$, as depicted in Fig. 1 . The seabed is considered time independent. The free surface, on the other hand, is time dependent. The lateral boundary, defined by $\Gamma_{n}$, is parallel to the $z$ direction and has a constant $(x, y)$ position. For simplicity, we do not consider open boundaries in this work. The domain boundary can thus be written as $\partial \Omega=\Gamma_{n} \cup \Gamma_{b} \cup \Gamma_{s}$. The unperturbed surface defined by $z=0$ is noted $\mathcal{T}$. We work within the scope of the Boussinesq and hydrostatic approximations.

\section{a. Equations and boundary conditions}

Let $\mathbf{u}(x, y, z, t)=[u(x, y, z, t) ; v(x, y, z, t)]$ be the horizontal velocity, with components $u$ and $v$ in the $x$ and $y$ directions, respectively. The vertical velocity is the component in the $z$ direction and is denoted by $w(x$, $y, z, t)$. The free-surface elevation $\eta(x, y, t)$ does not depend on $z$ and is defined with respect to the reference level $\mathcal{T}$. The unperturbed depth $d(x, y)$, also defined with respect to $\mathcal{T}$, is assumed to be time independent, does not depend on $z$, and is everywhere nonnegative. The layer thickness, $H$, is the sum of the depth and the free-surface elevation: $H(x, y, t)=d(x, y)+\eta(x, y, t)$. With those notations, the lower and upper domain boundaries are characterized by $\Gamma_{b} \equiv z=-d$ and $\Gamma_{s} \equiv$ $z=\eta$.

For the purpose of deriving the statements of volume and tracer conservation, there is no need to write out the full horizontal momentum equations. We simply assume that the horizontal velocity $\mathbf{u}$ is known in $\Omega(t)$ and that it satisfies the impermeability condition on the lateral boundary $\Gamma_{n}$; that is,

$$
\mathbf{u} \cdot \mathbf{n}=0 \quad \text { on } \quad \Gamma_{n},
$$

where $\mathbf{n}=\left(n_{x}, n_{y}\right)$ is the unit outward-pointing normal to $\Gamma_{n}$. We will also denote $n_{z}$ as the vertical component of the normal. In hydrostatic models, the vertical momentum equation reduces to hydrostatic equilibrium. Hence, there is no prognostic equation for the vertical velocity. The latter is computed diagnostically via the continuity equation:

$$
\boldsymbol{\nabla} \cdot \mathbf{u}+\frac{\partial w}{\partial z}=0 \text { in } \Omega,
$$

where $\boldsymbol{\nabla}$ is the horizontal gradient operator. Equation (2) is a statement of volume conservation. Another statement of volume conservation is obtained by depth integrating (2). This yields the following prognostic equation for the free-surface elevation:

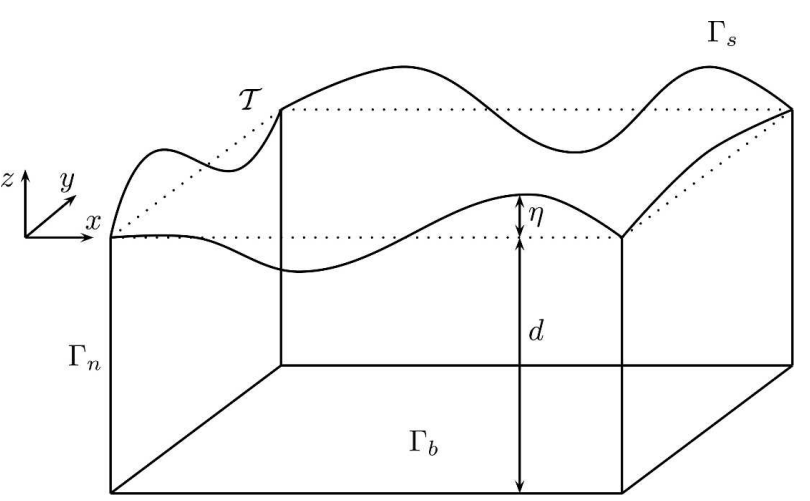

FIG. 1. Notation used to describe the three-dimensional timedependent domain $\Omega$. The seabed and the free surface are denoted by $\Gamma_{b}$ and $\Gamma_{s}$, respectively. The unperturbed plane defined by $z=0$ is noted $\mathcal{T}$ and is represented by the dotted lines. The lateral boundary is noted as $\Gamma_{n}$. At any location $(x, y)$, the depth $d(x, y)$ and the elevation $\eta(x, y, t)$ are both defined with reference to $\mathcal{T}$. The displacement of the free surface is exaggerated.

$$
\frac{\partial \eta}{\partial t}+\nabla \cdot \int_{-d}^{\eta} \mathbf{u} d z=q_{w} \text { on } \mathcal{T}
$$

for which use has been made of the impermeability of the seabed and the free surface. These impermeability conditions (the so-called kinematic boundary conditions) read

$$
\begin{aligned}
& w=-\mathbf{u} \cdot \nabla d \text { on } \Gamma_{b} \text { and } \\
& w=\frac{\partial \eta}{\partial t}+\mathbf{u} \cdot \nabla \eta-q_{w} \text { on } \Gamma_{s}
\end{aligned}
$$

where $q_{w}$ is the net freshwater volume flux per unit area (with units of a velocity) due to precipitation $\left(q_{w}>0\right)$, evaporation $\left(q_{w}<0\right)$, and river runoffs $\left(q_{w}>0\right)$, if the latter are not formulated as lateral boundary conditions. Note that boundary conditions (4) and (5) can also be written as

$$
\begin{aligned}
& \mathbf{u} \cdot \mathbf{n}+w n_{z}=0 \text { on } \Gamma_{b} \text { and } \\
& \mathbf{u} \cdot \mathbf{n}+w n_{z}=\left(\frac{\partial \eta}{\partial t}-q_{w}\right) n_{z} \text { on } \Gamma_{s},
\end{aligned}
$$

where we assume that the orientation of the freshwater flux is the same as that associated with the normal at the free surface. Finally, a given tracer with concentration $C$ obeys an advection-diffusion equation (with no source term) of the form

$$
\begin{aligned}
\frac{\partial C}{\partial t}+\boldsymbol{\nabla} \cdot( & \mathbf{u} C)+\frac{\partial(w C)}{\partial z} \\
& =\boldsymbol{\nabla} \cdot(\kappa \boldsymbol{\nabla} C)+\frac{\partial}{\partial z}\left(\kappa \frac{\partial C}{\partial z}\right) \text { in } \Omega,
\end{aligned}
$$


where $\kappa$ is the eddy diffusivity coefficient. A condition of no diffusive flux is prescribed at the boundary:

$$
\kappa \frac{\partial C}{\partial n}=0 \quad \text { on } \quad \partial \Omega,
$$

where $\partial C / \partial n$ is the normal derivative.

\section{b. Conservation properties}

From the equations presented above, we may now derive the statements of volume and tracer conservation and check the consistency between the tracer and continuity equations. For simplicity, the freshwater flux is set to zero $\left(q_{w}=0\right)$.

\section{1) Volume CONSERVATION}

By integrating (3) over the time-independent, twodimensional domain $\mathcal{T}$ and using the divergence theorem to compute the second integral, we obtain

$$
\int_{\mathcal{T}} \frac{\partial \eta}{\partial t} d \mathcal{T}+\int_{\Gamma_{n}} \mathbf{u} \cdot \mathbf{n} d \Gamma=0,
$$

which, by using the boundary condition (1), further reduces to

$$
\frac{d}{d t} \int_{\mathcal{T}} \eta \mathrm{d} \mathcal{T}=0
$$

Equation (10) is the statement of volume conservation.

\section{2) Global tracer conservation}

Integrating (8) over $\Omega(t)$, using the divergence theorem for the advection and diffusion terms, and enforcing the boundary condition (9) leads to

$$
\begin{aligned}
\int_{\Omega(t)} \frac{\partial C}{\partial t} \mathrm{~d} \Omega & +\int_{\Gamma_{n}} \mathbf{u} \cdot \mathbf{n} C d \Gamma+\int_{\Gamma_{b}}\left(\mathbf{u} \cdot \mathbf{n}+w n_{z}\right) C d \Gamma \\
& +\int_{\Gamma_{s}}\left(\mathbf{u} \cdot \mathbf{n}+w n_{z}\right) C d \Gamma=0 .
\end{aligned}
$$

The integrals over $\Gamma_{n}$ and $\Gamma_{b}$ vanish by enforcing boundary conditions (1) and (6). Using the Reynolds transport theorem, the first term of the expression above becomes

$$
\int_{\Omega(t)} \frac{\partial C}{\partial t} d \Omega=\frac{d}{d t} \int_{\Omega(t)} C d \Omega-\int_{\Gamma_{s}} C \frac{\partial \eta}{\partial t} n_{z} d \Gamma,
$$

with the velocity of the moving boundary $\Gamma_{s}$ being equal to that of the free surface. Equation (11) becomes

$$
\frac{d}{d t} \int_{\Omega(t)} C d \Omega+\int_{\Gamma_{s}}\left(\mathbf{u} \cdot \mathbf{n}+w n_{z}-\frac{\partial \eta}{\partial t} n_{z}\right) C d \Gamma=0 .
$$

Now, using boundary condition (7) yields the statement of global tracer conservation:

$$
\frac{d}{d t} \int_{\Omega(t)} C d \Omega=0 .
$$

\section{3) Consistency}

The property of consistency is equivalent to verifying that a constant tracer concentration is the solution to (8)-(9). Setting $C=C_{0} \neq 0$ in (8), with $C_{0}$ being a constant, we simply obtain

$$
C_{0}\left(\boldsymbol{\nabla} \cdot \mathbf{u}+\frac{\partial w}{\partial z}\right)=0 \quad \text { in } \quad \Omega .
$$

Therefore, the continuity and tracer equations are consistent (or compatible) with each other when the following relation holds true:

$$
\begin{aligned}
C_{0}\left(\boldsymbol{\nabla} \cdot \mathbf{u}+\frac{\partial w}{\partial z}\right) & =0 \Leftrightarrow \boldsymbol{\nabla} \cdot \mathbf{u}+\frac{\partial w}{\partial z} \\
& =0 \quad \text { in } \quad \Omega \text { and for } C_{0} \neq 0 .
\end{aligned}
$$

\section{The discrete conservation laws}

The purpose of this section is to derive the discrete counterparts of Eqs. (10), (12), and (13). We start by describing the mesh topology and introducing a few useful notations. We then present the variational formulations of the elevation, continuity, and tracer equations, followed by their discretization based upon the Galerkin procedure. The discrete conservation laws will then be inferred from the discrete equations. To keep notations more concise, all subsequent developments are carried out for the CG method.

\section{a. Mesh topology}

The numerical solution is sought in the threedimensional domain $\Omega^{h}$. The latter consists of an approximation of the physical domain, obtained by interpolating the boundaries of topographical features and the bathymetry. Within this framework, all boundaries are also interpolated so that we have $\partial \Omega \simeq \partial \Omega^{h}=\Gamma_{b}^{h} \cup$ $\Gamma_{s}^{h} \cup \Gamma_{n}^{h}$. The three-dimensional finite-element mesh (Fig. 2) is obtained by first partitioning $\mathcal{T}^{h}$ into $N_{t}$ open nonoverlapping triangles $\mathcal{T}_{e}$. Extrusion of each triangle $\mathcal{T}_{e}$ into a prismatic column is then performed so as to exactly fit the sea bottom $\Gamma_{b}^{h}$ and the free-surface $\Gamma_{s}^{h}$. The domain $\Omega^{h}$ is naturally partitioned by splitting up each column into prisms $\Omega_{e}$. 
$\mathcal{T}^{h}$

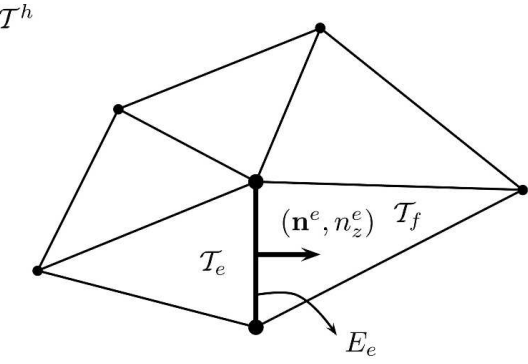

$\Omega^{h}$

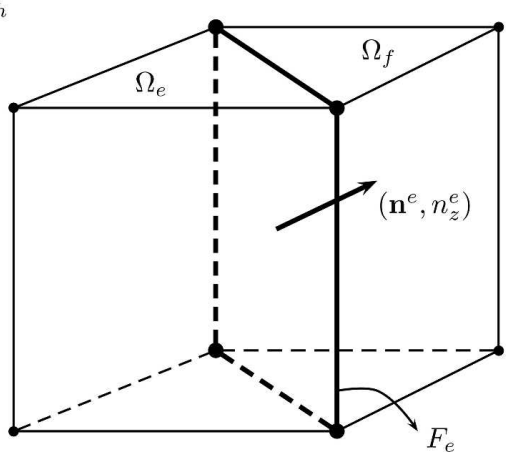

$\Omega^{h}$

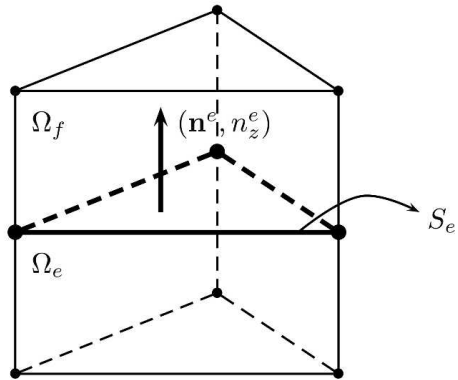

FIG. 2. The main notation used to describe the mesh topology. In two dimensions, any interior edge $E_{e}$ is shared by two triangles: $\mathcal{T}_{e}$ and $\mathcal{T}_{f}$. In three dimensions, any interior vertical face $F_{e}$ is common to the adjacent prisms $\Omega_{e}$ and $\Omega_{f}$ (lying within a common layer). Two stacked prisms, $\Omega_{e}$ and $\Omega_{f}$, share an interior triangular face, $S_{e}$. A unit-normal vector $\left(\mathbf{n}^{e}, n_{z}^{e}\right)$ is associated with each of these interior geometric items, with the superscript $e$ indicating that it is oriented from $e$ to $f$ (with $e>f$ ).

\section{b. Variational statements}

The variational statements involve integrations over geometrical items in two and three dimensions. The following notations are used:

$$
\begin{aligned}
& \int \square d \Omega, \quad 3 \mathrm{D} \text { integration over prisms; } \\
& \int \square d \Gamma, \quad \begin{array}{l}
\text { 2D integration over rectangular faces } \\
\text { (vertical faces); and }
\end{array} \\
& \int \square d \tau, \quad \text { 2D integration over triangular faces. }
\end{aligned}
$$

\section{1) Free-surface elevation equation}

For the free-surface equation [(3)], the variational statement at any given time $t$ consists in finding $\eta(x, y$, $t) \in \mathcal{H}$ such that

$$
\int_{\mathcal{T}^{h}}\left[\frac{\partial \eta}{\partial t}+\boldsymbol{\nabla} \cdot\left(\int_{-d}^{\eta} \mathbf{u} d z\right)\right] \hat{\eta} d \tau=0, \quad \forall \hat{\eta} \in \mathcal{H}
$$

where $\mathcal{H}$ is a suitable functional space. Integrating the divergence term by parts leads to

$$
\int_{\mathcal{T}^{h}}\left[\frac{\partial \eta}{\partial t} \hat{\eta}-\left(\int_{-d}^{\eta} \mathbf{u} d z\right) \cdot \boldsymbol{\nabla} \hat{\eta}\right] d \tau+\int_{\Gamma_{n}^{h}} \mathbf{u} \cdot \mathbf{n} \hat{\eta} d \Gamma=0
$$

$\forall \hat{\eta} \in \mathcal{H}$

By using the fact that $\nabla \hat{\eta}$ is independent of $z$ and by enforcing the impermeability condition (1), the above expression reduces to

$$
\int_{\mathcal{T}^{h}} \frac{\partial \eta}{\partial t} \hat{\eta} d \tau-\int_{\Omega^{h}} \mathbf{u} \cdot \nabla \hat{\eta} d \Omega=0, \quad \forall \hat{\eta} \in \mathcal{H}
$$

\section{2) Continuity equation}

For the continuity equation, the variational statement at any given time $t$ consists in finding $w(x, y, z, t) \in \mathcal{W}$ such that

$$
\int_{\Omega^{h}}\left(\boldsymbol{\nabla} \cdot \mathbf{u}+\frac{\partial w}{\partial z}\right) \hat{w} \mathrm{~d} \Omega=0, \quad \forall \hat{w} \in \mathcal{W}
$$


where $\mathcal{W}$ is another suitable functional space. By integrating the above expression by parts, we obtain

$$
\begin{aligned}
& -\int_{\Omega^{h}}\left(\mathbf{u} \cdot \boldsymbol{\nabla} \hat{w}+w \frac{\partial \hat{w}}{\partial z}\right) d \Omega+\underbrace{\int_{\Gamma_{n}^{h}} \hat{w} \mathbf{u} \cdot \mathbf{n} d \Gamma}_{1} \\
& +\underbrace{\int_{\Gamma_{b}^{h}} \hat{w}\left(\mathbf{u} \cdot \mathbf{n}+w n_{z}\right) d \tau}_{2}+\underbrace{\int_{\Gamma_{s}^{h}}^{\hat{w}\left(\mathbf{u} \cdot \mathbf{n}+w n_{z}\right) d \tau}}_{3}=0, \\
& \forall \hat{w} \in \mathcal{W} .
\end{aligned}
$$

A closer look at the terms labeled 1-3 will shed light on their meaning. By enforcing the impermeability condition of the lateral boundary and the seabed, namely, the conditions (1) and (6), integrals 1 and 2 vanish. Integral 3 does not vanish and must be computed in order to determine the vertical velocity on $\Gamma_{s}^{h}$. Note that the boundary condition (6) at the seabed is a natural boundary condition that is automatically incorporated into the variational statement. The continuity equation must then be integrated from the seabed upward. This can be done by using upwind-biased test functions for the $\mathrm{CG}$ method or upwind-biased fluxes for the DG method. In an intuitive interpretation, the continuity equation can be viewed as a steady-state advection equation (with the advective velocity equal to one) with a source term (the horizontal velocity divergence), which might help to clarify the approach described above. We end up with the following variational statement:

$$
\begin{aligned}
& -\int_{\Omega^{h}}\left(\mathbf{u} \cdot \nabla \hat{w}+w \frac{\partial \hat{w}}{\partial z}\right) d \Omega \\
& +\int_{\Gamma_{s}^{h}} \hat{w}\left(\mathbf{u} \cdot \mathbf{n}+w n_{z}\right) d \tau=0, \quad \forall \hat{w} \in \mathcal{W} .
\end{aligned}
$$

\section{3) Tracer equation}

We now turn our attention to the tracer equation [(8)]. Since the domain of integration $\Omega^{h}$ is time dependent and a time derivative appears in (8), a little more work is needed prior to deriving the variational statement. We will recast the original equation into the socalled arbitrary Lagrangian-Eulerian (ALE) form. With this formulation, the mesh is neither fixed in space, nor does it follow the fluid. It is therefore neither Eulerian nor Lagrangian. A good review of ALE methods is presented by Donea et al. (2004). Following Farhat et al. (2001), we define a reference fixed mesh $\Omega_{0}^{h}$ and a mapping function $\mathbf{x}$ between $\Omega_{0}^{h}$ and $\Omega^{h}$ :

$$
\mathcal{A}: \Omega_{0}^{h} \rightarrow \Omega^{h}: \boldsymbol{\xi} \rightarrow \mathcal{A}(\boldsymbol{\xi}, t)=\mathbf{x} .
$$

This mapping simply associates a three-dimensional coordinate $\boldsymbol{\xi}$ of the reference mesh $\Omega_{0}^{h}$ to a threedimensional coordinate $\mathbf{x}=(x, y, z)$ in the physical moving mesh $\Omega^{h}$. We further assume that this transformation is invertible:

$$
J=\left(\frac{\partial \mathbf{x}}{\partial \xi}\right)>0,
$$

where $J$ is the Jacobian of the transformation. We also require that the mapping associate the boundary of the reference mesh to the boundary of the physical mesh, that is, $\mathcal{A}\left(\partial \Omega_{0}^{h}\right)=\partial \Omega^{h}$, without any other constraint on the interior coordinates aside from some smoothness requirements. The conservative ALE form of (8) then reads (Formaggia and Nobile 2004)

$$
\begin{aligned}
\left.\frac{\partial(J C)}{\partial t}\right|_{\xi} & +J \boldsymbol{\nabla} \cdot(\mathbf{u} C)+J \frac{\partial(\tilde{w} C)}{\partial z} \\
& =J \boldsymbol{\nabla} \cdot(\kappa \boldsymbol{\nabla} C)+J \frac{\partial}{\partial z}\left(\kappa \frac{\partial C}{\partial z}\right) \text { in } \Omega_{0}^{h},
\end{aligned}
$$

where all terms are computed in the reference domain $\Omega_{0}^{h}$. In particular, the time derivative is computed with respect to a fixed position in $\Omega_{0}^{h}$. We have defined $\tilde{w}=$ $w-w_{m}$, with the mesh velocity $w_{m}$ given by

$$
w_{m}=\left.\frac{\partial z}{\partial t}\right|_{\xi} \text {. }
$$

With the presence of the mesh velocity, vertical advection is taken relative to the moving mesh. The variational statement for the tracer equation is obtained by multiplying (18) by a test function $\hat{C}$, integrating the result over the reference mesh and using the fact that $d \Omega=J d \Omega_{0}$. We seek $C \in \mathcal{G}$, such that

$$
\begin{aligned}
& \frac{d}{d t} \int_{\Omega^{h}(t)} C \hat{C} d \Omega+\int_{\Omega^{h_{(t)}}}\left[\boldsymbol{\nabla} \cdot(\mathbf{u} C)+\frac{\partial(\tilde{w} C)}{\partial z}\right] \hat{C} d \Omega \\
& \quad=\int_{\Omega^{h(t)}}\left[\boldsymbol{\nabla} \cdot(\kappa \nabla C)+\frac{\partial}{\partial z}\left(\kappa \frac{\partial C}{\partial z}\right)\right] \hat{C} d \Omega, \quad \forall \hat{C} \in \mathcal{G},
\end{aligned}
$$

where $\mathcal{G}$ is an appropriate functional space. The first term in the above expression was obtained by using the following result:

$$
\begin{aligned}
\left.\int_{\Omega_{0}^{h}} \frac{\partial(J C)}{\partial t}\right|_{\xi} \hat{C} d \Omega_{0} & =\frac{d}{d t} \int_{\Omega_{0}^{h}} J C \hat{C} d \Omega_{0} \\
& =\frac{d}{d t} \int_{\Omega^{h^{h}}(t)} C \hat{C} d \Omega,
\end{aligned}
$$


where we used the fact that the test function does not depend on time in the reference mesh. It does, however, depend on time in the physical domain $\Omega^{h}$. The advection and diffusion terms can be integrated by parts, which yields

$$
\begin{aligned}
\int_{\Omega^{h}(t)}[\boldsymbol{\nabla} \cdot(\mathbf{u} C) & \left.+\frac{\partial(\tilde{w} C)}{\partial z}\right] \hat{C} d \Omega \\
= & -\int_{\Omega^{h}(t)} C\left(\mathbf{u} \cdot \boldsymbol{\nabla} \hat{C}+\tilde{w} \frac{\partial \hat{C}}{\partial z}\right) d \Omega \\
& +\int_{\Gamma_{s}^{h}} C \hat{C}\left(\mathbf{u} \cdot \mathbf{n}+\tilde{w} n_{z}\right) d \tau
\end{aligned}
$$

and

$$
\begin{aligned}
\int_{\Omega^{h}(t)}[\boldsymbol{\nabla} & \left.\cdot(\kappa \nabla C)+\frac{\partial}{\partial z}\left(\kappa \frac{\partial C}{\partial z}\right)\right] \hat{C} d \Omega \\
& =-\int_{\Omega^{h_{(t)}}} \kappa\left(\boldsymbol{\nabla} \hat{C} \cdot \nabla C+\frac{\partial \hat{C}}{\partial z} \frac{\partial C}{\partial z}\right) d \Omega,
\end{aligned}
$$

where we used the no-flux conditions (1), (6), and (9). Note that the right-hand side of (20) is nothing but the variational statement of the continuity equation [see (17)] in which the velocity is multiplied by the tracer concentration $C$, the test function $\hat{w}$ is replaced by $\hat{C}$, and the modified vertical velocity $\tilde{w}$ is used in place of the vertical velocity $w$. Now, using (20) and (21) in (19) gives the following:

$$
\begin{aligned}
& \frac{d}{d t} \int_{\Omega^{h}(t)} C \hat{C} d \Omega-\int_{\Omega^{h}(t)} C\left(\mathbf{u} \cdot \nabla \hat{C}+\tilde{w} \frac{\partial \hat{C}}{\partial z}\right) d \Omega \\
& +\int_{\Gamma_{s}^{h}} C \hat{C}\left(\mathbf{u} \cdot \mathbf{n}+\tilde{w} n_{z}\right) d \tau \\
& +\int_{\Omega^{h}(t)} \kappa\left(\nabla \hat{C} \cdot \nabla C+\frac{\partial \hat{C}}{\partial z} \frac{\partial C}{\partial z}\right) d \Omega=0, \quad \forall \hat{C} \in \mathcal{G} .
\end{aligned}
$$

\section{c. Finite-element discretization}

We now seek approximations $\eta^{h} \simeq \eta, w^{h} \simeq w$, and $C^{h} \simeq C$ in finite-dimensional subsets of $\mathcal{H}, \mathcal{W}$, and $\mathcal{G}$, respectively. Each of those subsets, marked by a superscript $h$, is spanned by a finite number of polynomial basis functions. The basis functions will be noted $\psi$ with a superscript indicating the variable to which it is associated. The finite-element approximations are

$$
\begin{aligned}
\eta \simeq \eta^{h} & =\sum_{j=1}^{N_{\eta}} H_{j}(t) \psi_{j}^{\eta}(x, y) \in \mathcal{H}^{h} \\
& =\operatorname{span}\left\{\psi_{1}^{\eta}, \psi_{2}^{\eta}, \ldots, \psi_{N_{\eta}}^{\eta}\right\} \subset \mathcal{H}, \\
w \simeq w^{h} & =\sum_{j=1}^{N_{w}} W_{j}(t) \psi_{j}^{w}(x, y, z) \in \mathcal{W}^{h} \\
& =\operatorname{span}\left\{\psi_{1}^{w}, \psi_{2}^{w}, \ldots, \psi_{N_{w}}^{w}\right\} \subset \mathcal{W}, \\
C \simeq C^{h} & =\sum_{j=1}^{N_{C}} C_{j}(t) \psi_{j}^{C}(x, y, z) \in \mathcal{G}^{h} \\
& =\operatorname{span}\left\{\psi_{1}^{C}, \psi_{2}^{C}, \ldots, \psi_{N_{C}}^{C}\right\} \subset \mathcal{G} .
\end{aligned}
$$

Note that, strictly speaking, the three-dimensional basis functions depend on time because of the mesh motions. This, however, only has an implication on the tracer equation where a time derivative appears. We now opt for the Galerkin method, which is equivalent to the following procedure. Consider each variational statement, (15), (17), and (22), in which the sought variable ( $\eta, w$, and $C$, respectively) is substituted for its approximation ( $\eta^{h}, w^{h}$, and $C^{h}$, respectively) and hold it true when the test function is substituted for any of the basis functions spanning the corresponding subset to which the test function belongs. We also assume that we possess an approximation $\mathbf{u}^{h}$ of the horizontal velocity field, the obtainment of which is beyond the scope of this paper [see White (2007) instead].

The discrete variational statement for the elevation, (15), now consists in finding $\eta^{h} \in \mathcal{H}^{h}$, such that

$$
\begin{aligned}
\int_{\mathcal{T}^{h}} \frac{\partial \eta^{h}}{\partial t} \psi_{i}^{\eta} d \tau-\int_{\Omega^{h}} \mathbf{u}^{h} \cdot \nabla \psi_{i}^{\eta} d \Omega & =0, \\
\forall i & =1,2, \ldots, N_{\eta} .
\end{aligned}
$$

For the continuity equation, the discrete variational statement consists in finding $w^{h} \in \mathcal{W}^{h}$, such that

$$
\begin{aligned}
& -\int_{\Omega^{h}}\left(\mathbf{u}^{h} \cdot \nabla \psi_{i}^{w}+w^{h} \frac{\partial \psi_{i}^{w}}{\partial z}\right) d \Omega \\
& +\int_{\Gamma_{s}^{h}} \psi_{i}^{w}\left(\mathbf{u}^{h} \cdot \mathbf{n}+w^{h} n_{z}\right) d \tau=0, \quad \forall i=1,2, \ldots, N_{w} .
\end{aligned}
$$

Last, the discrete variational statement for the tracer equation consists in finding $C^{h} \in \mathcal{G}^{h}$, such that 


$$
\begin{array}{r}
\frac{d}{d t} \int_{\Omega^{h_{(t)}}} C^{h} \psi_{i}^{C} d \Omega-\int_{\Omega^{h_{(t)}}} C^{h}\left(\mathbf{u}^{h} \cdot \nabla \psi_{i}^{C}+\tilde{w}^{h} \frac{\partial \psi_{i}^{C}}{\partial z}\right) d \Omega \\
+\int_{\Gamma_{s}^{h}} C^{h} \psi_{i}^{C}\left(\mathbf{u}^{h} \cdot \mathbf{n}+\tilde{w}^{h} n_{z}\right) d \tau \\
+\int_{\Omega^{h_{(t)}}} \kappa\left(\nabla \psi_{i}^{C} \cdot \nabla C^{h}+\frac{\partial \psi_{i}^{C}}{\partial z} \frac{\partial C^{h}}{\partial z}\right) d \Omega=0, \\
\forall i=1,2, \ldots, N_{C} .
\end{array}
$$

\section{d. Discrete conservation laws}

Starting from the discrete variational statements in (24)-(26), we now investigate under which conditions the global volume and tracer conservation, as well as consistency (i.e., compatibility), are achieved in the discrete sense.

\section{1) Volume CONSERVATION}

Since the variational formulation (24) must be valid for any $\psi_{i}^{\eta}$, it must hold true for $\psi_{i}^{\eta}=1$ as well (which belongs to $\mathcal{H}^{h}$ ). Setting $\psi_{i}^{\eta}=1$ in (24) and using the fact that $\mathcal{T}^{h}$ is time independent gives

$$
\frac{d}{d t} \int_{\mathcal{T}^{h}} \eta^{h} d \tau=0
$$

which is the discrete statement of volume conservation.

\section{2) Global tracer conservation}

The properties of global tracer conservation are investigated by setting $\psi_{i}^{C}=1$ in the discrete variational statement for the tracer equation [(26)]. We then obtain

$$
\frac{d}{d t} \int_{\Omega^{h}(t)} C^{h} d \Omega+\int_{\Gamma_{s}^{h}} C^{h}\left[\mathbf{u}^{h} \cdot \mathbf{n}+\left(w^{h}-w_{m}^{h}\right) n_{z}\right] d \tau=0,
$$

where we used $\tilde{w}^{h}=w^{h}-w_{m}^{h}$. In view of (28), providing that the integral over the free surface $\Gamma_{s}^{h}$ is discarded, the tracer is globally conserved; namely,

$$
\frac{d}{d t} \int_{\Omega^{h}(t)} C^{h} d \Omega=0 .
$$

However, discarding this integral consistently - that is, while preserving the discrete compatibility between the elevation, continuity, and tracer equations-brings about additional constraints as we will now see.

\section{3) COnSistency}

According to the definition presented earlier, consistency is equivalent to requiring that a constant concentration be solution to (26). Setting $C^{h}=C_{0} \neq 0$ in (26), factoring out $C_{0}$, and separating out the terms depending on the mesh velocity (and integrating them by parts) from those that do not, we end up having to satisfy the following equation:

$$
\begin{aligned}
& \underbrace{\frac{d}{d t} \int_{\Omega^{h}(t)} \psi_{i}^{C} d \Omega}_{A_{1}}-\underbrace{\int_{\Omega^{h}(t)} \psi_{i}^{C} \frac{\partial w_{m}^{h}}{\partial z} d \Omega}_{A_{2}} \\
&-\underbrace{\int_{\Omega^{h}(t)}\left(\mathbf{u}^{h} \cdot \nabla_{\psi_{i}^{C}}+w^{h} \frac{\partial \psi_{i}^{C}}{\partial z}\right) d \Omega}_{B_{1}} \\
&+\underbrace{\int_{\Gamma_{s}}}_{B_{2} \psi_{i}^{C}\left(\mathbf{u}^{h} \cdot \mathbf{n}+w^{h} n_{z}\right) d \tau}=0, \\
& \forall i=1,2, \ldots, N_{C} .
\end{aligned}
$$

The term labeled $A_{2}$ is the result of integrating by parts all terms involving the mesh velocity. The set of $B$ labeled terms in the above expression and the discrete variational statement for the continuity equation [(25)] are identical under the following two conditions:

1) the subsets $\mathcal{W}^{h}$ and $\mathcal{G}^{h}$ are the same (in which case $\left.\psi_{i}^{w}=\psi_{i}^{C} \forall i=1,2, \ldots N^{w}=N^{C}\right)$ and

2) the $B$-labeled terms and (25) are computed on the same mesh, $\Omega^{h}(t)$.

These two conditions may be summarized by simply demanding that (25) and the advection terms in (30) be discretely compatible; a condition that we will call discrete compatibility between the continuity and tracer equations. Concretely, this entails that the same elements must be used to compute $w^{h}$ and $C^{h}$. Note that this condition is conceptually the same as that derived when using finite differences (Griffies et al. 2001). Now, the fulfillment of this condition does not necessarily ensure that (30) is satisfied. The following relation between the $A$-labeled terms in (30) must also hold true:

$$
\frac{d}{d t} \int_{\Omega^{h}(t)} \psi_{i}^{C} d \Omega=\int_{\Omega^{h}(t)} \psi_{i}^{C} \frac{\partial w_{m}^{h}}{\partial z} d \Omega
$$

When using a discontinuous representation, the above expression must be true for each element $\Omega_{e}(t)$ individually when $\psi_{i}^{C}=1$, which is a stricter condition. 
When discretized in time and when $\psi_{i}^{C}=1$, (31) becomes

$\operatorname{VOL}\left(\Omega_{e}^{n+1}\right)-\operatorname{VOL}\left(\Omega_{e}^{n}\right)=\int_{t^{n}}^{t^{n+1}}\left(\int_{\Omega_{e}(t)} \frac{\partial w_{m}^{h}}{\partial z} d \Omega\right) d t$

where the time step shall be defined as $\Delta t=t^{n+1}-t^{n}$. Equation (32) is known as the discrete geometric conservation law (DGCL; Farhat et al. 2001; Donea et al. 2004; Formaggia and Nobile 2004) and states that the variation in volume of a given element over $\Delta t$ must be equal to the volume swept by the element boundaries (with velocity $w_{m}$ ) during that time interval. Note that if the volume is computed exactly, then the time integration on the right-hand side of (32) must be exact. Depending upon the hypotheses made regarding the time dependence of $w_{m}^{h}$, a proper quadrature rule must then be used. Hence, to ensure consistency, the following two conditions, at least, must be fulfilled:

1) the continuity and tracer equations must be discretely compatible (i.e., the way we compute the vertical velocity must be discretely compatible with the way we compute the advection terms in the tracer equation) and

2) the mesh update procedure must comply with the DGCL [see (32)].

It should be emphasized that these conditions involve the continuity and tracer equations as well as the mesh update procedure. So far, nothing has been said regarding the free-surface elevation equation.

Conserving the tracer globally while preserving consistency between the continuity and tracer equations requires that the surface integral on $\Gamma_{s}^{h}$ automatically vanish when $C^{h}=1$ in (28). This will happen only if the computation of the vertical velocity guarantees it. We now verify that this is the case. Let us return to the continuity equation and focus on the discrete variational statement $[(25)]$. Since the mesh is made up of prisms with vertical faces, we can readily add up the components of (25) written for those $\psi_{i}^{w}$ sharing the same two-dimensional support. We note that $I$ is the set of indices $i$ corresponding to the basis functions $\psi_{i}^{w}$ aligned on the same vertical. By definition, all of these basis functions satisfy the following two properties:

$$
\begin{aligned}
\sum_{i \in I} \psi_{i}^{w}(x, y, z) & =\psi_{I}^{w, 2 D}(x, y), \\
\sum_{i \in I} \frac{\partial \psi_{i}^{w}}{\partial z}(x, y, z) & =0,
\end{aligned}
$$

where $\psi_{I}^{w, 2 D}$ is simply the projection of $\psi_{i}^{w}(i \in I)$ onto the plane $(x, y)$. This projection is identified by $I$. The first summation represents the fact that the vertical dependences of all basis functions cancel out. Now, adding up the components of (25) in the vertical gives rise to

$$
-\int_{\Omega^{h}} \mathbf{u}^{h} \cdot \nabla \psi_{I}^{w, 2 D} d \Omega+\int_{\Gamma_{s}^{h}} \psi_{I}^{w, 2 D}\left(\mathbf{u}^{h} \cdot \mathbf{n}+w^{h} n_{z}\right) d \tau=0 .
$$

The similarities between the above expression and the discrete variational statement for the elevation [(24)] are clear. By choosing the basis functions for the vertical velocity such that $\psi_{I}^{w, 2 D}=\psi_{I}^{\eta}$, where $\psi_{I}^{\eta}$ is the twodimensional elevation basis function, the second term in the discrete elevation equation $[(24)]$ is identical to the first term in (33). This leaves us with the following equality:

$$
\begin{aligned}
\int_{\Gamma_{s}^{h}} \psi_{I}^{w, 2 D}\left(\mathbf{u}^{h} \cdot \mathbf{n}+w^{h} n_{z}\right) d \tau & =\int_{T^{h}} \psi_{I}^{\eta} \frac{\partial \eta^{h}}{\partial t} d \tau \\
& =\int_{\Gamma_{s}^{h}} \psi_{I}^{\eta} \frac{\partial \eta^{h}}{\partial t} n_{z} d \tau,
\end{aligned}
$$

where $n_{z}$ is the Jacobian of the transformation of coordinates from $\mathcal{T}^{h}$ to $\Gamma_{s}^{h}$. The last expression thus becomes

$$
\int_{\Gamma_{s}^{h}} \psi_{i}^{w}\left[\mathbf{u}^{h} \cdot \mathbf{n}+\left(w^{h}-\frac{\partial \eta^{h}}{\partial t}\right) n_{z}\right] d \tau=0,
$$

where we used the fact that $\psi_{i}^{w}$ reduces to its twodimensional structure on $\Gamma_{s}^{h}$. Equation (35) is the discrete counterpart of the kinematic boundary condition on the vertical velocity, given by (7). Most importantly, this result demonstrates that global conservation can be achieved without breaking down consistency. When setting $C^{h}=1$ in (26), the surface integral on $\Gamma_{s}^{h}$ vanishes if the mesh velocity at the sea surface is $w_{m}^{h}=$ $\partial \eta^{h} / \partial t$ and the same interpolation is used in the horizontal for the elevation and the vertical velocity. Under these two conditions, the surface integral can be discarded consistently in (28).

The discrete surface kinematic boundary condition is retrieved by adding up the discrete components of the continuity equation in the vertical. This is a consequence of the elevation and continuity equations being discretely compatible. And they should be since they express the same principle of volume conservation. It then turns out that the surface kinematic boundary condition is redundant, complying with the first-order nature of the continuity equation. In the appendix, we 
show that integrating the continuity equation downward (with the imposition of the surface boundary condition) allows for automatically retrieving the seabed boundary condition, provided of course that the continuity and elevation equations are discretely compatible. Both directions of integration yield equivalent results (the correct boundary condition is retrieved) and the tracer conservation does not depend on it (the tracer is consistently conserved in both cases). It is easy to conjure up a way of breaking down consistency while preserving global conservation. By enforcing the surface tracer flux to vanish, global conservation is ensured. However, if there is no compatibility between the elevation and the continuity equations, this surface integral does not naturally vanish and consistency breaks down. Now, an opposite scenario can be imagined. Consistency is easily achieved by ensuring that the tracer and continuity equations are discretely compatible. Nevertheless, if the surface integral does not vanish and is computed (to ensure consistency), global conservation will break down for a tracer distribution different than a constant value throughout the domain.

The key results regarding conservation are summarized below. In the absence of source terms and diffusive boundary fluxes, the following are sufficient conditions to consistently achieve global tracer conservation:

1) The continuity and tracer equations are discretely compatible, which comes down to having the discrete advection terms in the tracer equation reduce to the discrete continuity equation when $C^{h}=1$ [see (30)].

2) The DGCL is satisfied elementwise [see (32)].

3) The elevation and continuity equations are discretely compatible. This condition entails that the discrete surface kinematic boundary condition is retrieved when adding up all components of the discrete continuity equation in the vertical [see (35)].

4) The mesh velocity at the surface $\Gamma_{s}^{h}$ is oriented in the $z$ direction and has a magnitude of $w_{m}^{h}=\partial \eta^{h} / \partial t$. Note that, in the interior, the mesh motion is not constrained unless element shape regularity requires it.

\section{e. Which elements should we use?}

The above conditions restrict the choice of finiteelement subsets and, thereby, the choice of elements that can be used. These restrictions lead to the following guidelines: (i) The same element must be used for the vertical velocity and the tracer. (ii) The nodes locations in the horizontal must be the same for the el- evation and the vertical velocity. (iii) The two previous statements also imply that the nodes locations in the horizontal must be the same for the elevation, the vertical velocity, and the tracer. (iv) In the vertical, the nodes locations for the vertical velocity and the tracer is unconstrained, yet must be identical for both variables.

In addition to these considerations, the mixed formulation used for the horizontal velocity and the elevation must be numerically stable. The mixed formulation should be devoid of spurious elevation and velocity modes. In the presence of spurious elevation modes, a stabilized formulation can usually filter out the modes. However, this requires the addition of a term to the discrete elevation equation, with the consequence of breaking down the discrete compatibility between the elevation and continuity equations. Velocity modes are less problematic insofar as a small amount of momentum diffusion is usually sufficient to filter them out. Based on the most recent studies, the two mixed formulations that turn out to be the most effective for hydrostatic ocean modeling based on the primitive equations are the $P_{1}^{\mathrm{NC}}-P_{1}$ and the $R T_{0}$ pairs (Hanert et al. 2003; Le Roux 2005; Le Roux et al. 2005, 2007). The first pair was originally used by Hua and Thomasset (1984) for shallow-water flows and consists of a linear nonconforming interpolation for both components of the velocity and a linear interpolation for the elevation. It does not support any spurious oscillations. This pair has been used by Hanert et al. (2005) and White et al. (2006a) in two dimensions and by White and Deleersnijder (2007) in three dimensions. The second pair is called a low-order Raviart-Thomas element. The normal velocity components are located at the middle of each side of the triangular element and the elevation is constant on each element. Shallow-water models using this formulation are described by Miglio et al. (1998) and Walters (2005).

Using the $P_{1}^{\mathrm{NC}}-P_{1}$ pair requires opting for a $P_{1}$ representation in the horizontal for the tracer and the vertical velocity (in order to consistently ensure global conservation). To fulfill the same property with the $R T_{0}$ element, the vertical velocity and the tracer must be constant in the horizontal on each element. In both cases, there is no constraint for the vertical interpolation: it could be of high order and discontinuous. Both schemes have advantages and disadvantages. A linear continuous representation for the tracers is not optimal for advection-dominated flows. Stabilization could be necessary (without any impact on conservation). The finite-volume scheme pertaining to the $R T_{0}$ element is certainly more stable but might be overdiffusive. Regarding the elevation, $P_{1}$ is more accurate than $P_{0}$ with 
the latter leading to twice as many degrees of freedom. Moreover, a $P_{1}$ interpolation for the elevation leads to a piecewise linear (and continuous) representation of the moving mesh. Finally, when using the $P_{1}^{\mathrm{NC}}-P_{1}$ pair, to be computationally competitive, the matrices of the systems for computing the vertical velocity and the tracer must be rendered as at least banded diagonal through mass lumping.

\section{f. The issue of time stepping}

In light of the developments presented out above, time discretization does not appear to be an issue. However, there is more involved with conservation and consistency than the proper choice of elements for spatial discretization. In the following discussion, we present a time-stepping algorithm that ensures conservation and consistency in cases of both nonlinear and linearized free-surface equations. We first assume that the freesurface elevation equation and the three-dimensional horizontal momentum equation are solved simultaneously. We then opt for a mode-splitting approach for which we still assume that the same time step is employed for both modes but a sequential approach is used. The free-surface elevation equation is first solved, followed by the three-dimensional momentum equation. In this context, we then show how to satisfy conservation and consistency when using a linearized freesurface elevation equation. Finally, we touch upon the issue of using different time steps.

\section{1) Simultaneous Resolution}

Starting from (24) - in which the superscript $h$ has been dropped for clarity and replaced by the time index instead-a generic, fully discrete, free-surface elevation equation is given by

$$
\int_{\mathcal{T}} \frac{\eta^{n+1}-\eta^{n}}{\Delta t} \psi_{i}^{\eta} d \tau=\int_{\Omega^{n}} \mathbf{u}^{n+\theta} \cdot \nabla \psi_{i}^{\eta} d \Omega
$$

where $\mathbf{u}^{n+\theta}=\theta \mathbf{u}^{n+1}+(1-\theta) \mathbf{u}^{n}$, with $0.5 \leq \theta \leq 1$ to yield an implicit scheme and circumvent the stability constraint due to inertia-gravity waves. Evaluating the right-hand side integral on mesh $\Omega^{n}$ amounts to linearizing in time the free-surface elevation equation and is performed for convenience and to avoid having to solve a nonlinear equation. To preserve the discrete compatibility between the free-surface and continuity equations, we must ensure that the right-hand side of (36) is retrieved by adding up the components of the continuity equation in the vertical, as was done in (33) and (34). Doing so on a mesh at time step $n$ gives rise to

$$
\begin{aligned}
\int_{\Gamma_{s}^{n}} \psi_{I}^{w, 2 D}\left(\mathbf{u}^{n+\theta} \cdot \mathbf{n}+w^{n+\theta} n_{z}\right) d \tau \\
=\int_{\Omega^{n}} \mathbf{u}^{n+\theta} \cdot \nabla \psi_{I}^{w, 2 D} d \Omega,
\end{aligned}
$$

which is the time-discretized equivalent of (33). For the consistent spatial scheme outlined in section $3 \mathrm{~d}$, discrete compatibility between the fully discrete elevation and continuity equations is achieved provided that the same time-discretized horizontal velocity is employed in the right-hand sides of (36) and (37), which is the choice made here. In that case, we obtain

$$
\int_{\Gamma_{s}^{n}} \psi_{i}^{w}\left[\mathbf{u}^{n+\theta} \cdot \mathbf{n}+\left(w^{n+\theta}-\frac{\eta^{n+1}-\eta^{n}}{\Delta t}\right) n_{z}\right] d \tau=0
$$

which is the time-discretized version of (35) or the fully discrete version of the surface kinematic boundary condition (7).

Given that the continuity equation is discretized on the mesh at time step $n$, advection terms in the tracer equation must be discretized on this same mesh, giving rise to the following fully discrete tracer equation (from which diffusion has been dropped for simplicity):

$$
\begin{aligned}
& \frac{1}{\Delta t}\left(\int_{\Omega^{n+1}} C^{n+1} \psi_{i}^{C} d \Omega-\int_{\Omega^{n}} C^{n} \psi_{i}^{C} d \Omega\right) \\
& -\int_{\Omega^{n}} C^{n}\left[\mathbf{u}^{n+\theta} \cdot \nabla \psi_{i}^{C}+\left(w^{n+\theta}-w_{m}^{n+1}\right) \frac{\partial \psi_{i}^{C}}{\partial z}\right] d \Omega=0 .
\end{aligned}
$$

The surface boundary term in the above equation has been discarded to ensure global conservation. This latter property is readily achieved by choosing $\psi_{i}^{C}=1$. Moreover, when setting $C^{n}$ to 1 , this surface integral reduces to

$$
\int_{\Gamma_{s}^{n}} \psi_{i}^{C}\left[\mathbf{u}^{n+\theta} \cdot \mathbf{n}+\left(w^{n+\theta}-\frac{\eta^{n+1}-\eta^{n}}{\Delta t}\right) n_{z}\right] d \tau
$$

which simply is the fully discrete surface kinematic boundary condition (38), retrieved if $\psi_{i}^{C}=\psi_{i}^{w}$. Hence, the surface integral can be discarded while preserving consistency. Finally, a constant tracer concentration is a solution to (39) if the DGCL is satisfied elementwise and tracer advection is discretely compatible with the continuity equation. These two requirements are actually met by the spatial discretization, as presented in 
section 3d. As a consequence, the fully discrete scheme presented above is conservative, namely,

$$
\int_{\Omega^{n+1}} C^{n+1} d \Omega=\int_{\Omega^{n}} C^{n} d \Omega
$$

and locally consistent. At this point, it is worth making three comments. (i) In (39), the tracer concentration $C^{n}$ was taken to be explicit in time; it could have been treated implicitly in time without consequences given the conclusions made about conservation and consistency. (ii) The mesh velocity is given a time step $n+1$ because it is computed after knowing the new elevation field. (iii) The fully discrete algorithm is staggered in time, providing the elevation and the tracers at integer time steps and the full velocity at intermediate time steps $n+\theta$, where $\theta$ is commonly taken to be 0.5 to yield a semi-implicit scheme, in which case the elevation equation must be properly coupled with the horizontal momentum equation.

\section{2) Mode SPlitting Resolution}

For reasons of computational performance, the dynamics are usually split into external and internal modes (e.g., Simons 1974; Blumberg and Mellor 1987; Killworth et al. 1991). The external mode is governed by a depth-integrated, two-dimensional system, while the internal mode is governed by the three-dimensional equations. The dynamics of the external mode includes fast-propagating inertia-gravity waves while the dynamics of the internal mode is much slower. The split allows for treating the external mode semi-implicitly in time at a reasonable cost or explicitly with time steps smaller than those used to advance the internal mode. In the first approach, the same time step can still be used to advance all equations. In the second approach, two different time steps are used. In both cases, the effect is a filtering of the undesired fast surface waves.

Here, we make the choice to use the same time step. Since the external mode equations are two-dimensional, the elevation equation is given by

$$
\int_{\mathcal{T}} \frac{\eta^{n+1}-\eta^{n}}{\Delta t} \psi_{i}^{\eta} d \tau=\int_{\mathcal{T}} H^{n} \overline{\mathbf{u}}^{n+\theta} \cdot \nabla \psi_{i}^{\eta} d \tau,
$$

where $\overline{\mathbf{u}}$ is the depth-averaged horizontal velocity, $H^{n}=$ $d+\eta^{n}$ is the total depth, and $H^{n} \overline{\mathbf{u}}$ is the horizontal barotropic transport. It is well known that this splitting introduces a discrepancy between the transport associated with the two-dimensional dynamics and that associated with the three-dimensional dynamics. This is so because the mode separation is, in general, inexact
(Killworth et al. 1991; Higdon and de Szoeke 1997; Griffies et al. 2001). Because of this discrepancy, the discrete compatibility between the elevation and continuity equations may break down. In general, we have

$$
\int_{\mathcal{T}} H^{n} \overline{\mathbf{u}}^{n+\theta} \cdot \nabla \psi_{i}^{\eta} d \tau \neq \int_{\Omega^{n}} \mathbf{u}^{n+\theta} \cdot \nabla \psi_{i}^{\eta} d \Omega,
$$

which explains the loss of compatibility. One way of reconciling this discrepancy-and recovering the discrete compatibility between the elevation and continuity equations - is to ensure that the transport associated with the full three-dimensional horizontal velocity $\mathbf{u}$ is equal to the transport associated with the depthaveraged horizontal velocity $\overline{\mathbf{u}}$. The procedure consists of defining a modified horizontal velocity $\mathbf{u}_{*}^{n+\theta}$ such that, for a fixed position in the horizontal plane, we have

$$
\int_{-d}^{\eta^{n}} \mathbf{u}_{*}^{n+\theta} d z=H^{n} \overline{\mathbf{u}}^{n+\theta}
$$

The modified horizontal velocity is defined as

$$
\mathbf{u}_{*}^{n+\theta}=\mathbf{u}^{n+\theta}-\frac{1}{H^{n}} \int_{-d}^{\eta^{n}} \mathbf{u}^{n+\theta} d z+\overline{\mathbf{u}}^{n+\theta} .
$$

It is worth pointing out again that this correction is necessary because of the splitting between the external and internal modes. The latter is made to improve the computational performance of the algorithm and the price to pay for this improvement is the discrepancy. However, because conservation and consistency are properties of such great importance, this discrepancy must be reconciled.

\section{3) The Linearized free-Surface equation}

For large-scale ocean simulations over climatic time scales, it is common to assume that $\eta \ll d$ so that the free-surface elevation equation can be linearized in space. This further improves the computational performance because the system matrix associated with (40) does not have to be rebuilt at each time step. In that case, the free-surface elevation equation simply becomes

$$
\int_{\mathcal{T}} \frac{\eta^{n+1}-\eta^{n}}{\Delta t} \psi_{i}^{\eta} d \tau=\int_{\mathcal{T}} d \overline{\mathbf{u}}^{n+\theta} \cdot \nabla \psi_{i}^{\eta} d \tau .
$$

This approximation does not imply that conservation and consistency break down and that the threedimensional mesh no longer moves, as was earlier suggested (Roullet and Madec 2000). Once the elevation $\eta^{n+1}$ is computed according to (42), the computational 
domain is deformed in compliance with this new elevation field. The three-dimensional horizontal velocity $\mathbf{u}^{n+\theta}$ is computed on the mesh $\Omega^{n}$ and its correction must take into account the fact that we use a linearized free-surface elevation equation. Hence, $\mathbf{u}_{*}^{n+\theta}$ is given by

$$
\mathbf{u}_{*}^{n+\theta}=\mathbf{u}^{n+\theta}-\frac{1}{H^{n}} \int_{-d}^{\eta^{n}} \mathbf{u}^{n+\theta} d z+\frac{d}{H^{n}} \overline{\mathbf{u}}^{n+\theta},
$$

which is to be compared with (41). When depth integrated, (43) becomes

$$
\int_{-d}^{\eta^{n}} \mathbf{u}_{*}^{n+\theta} d z=d \overline{\mathbf{u}}^{n+\theta},
$$

which renders the continuity equation compatible with the linearized free-surface elevation [(42)]. Note that (43) is very similar to (41) except for the factor $d / H^{n}$ before the last term. In most large-scale applications, this factor is close to 1 but must be introduced to yield conservation and consistency to machine accuracy. Indeed, this correction ensures compatibility between the free-surface and continuity equations, which, in turn, ensures global tracer conservation in a consistent way. Again, the trade-off is the correction step versus an efficient algorithm. When we opt for mode splitting, in cases of both nonlinear and linearized free-surface elevation equations, the vertical velocity $w^{n+\theta}$ is computed using the modified horizontal velocity in (41) or (43), depending on the context.

\section{4) AN EXPLiCit EXTERNAL MODE}

In the foregoing discussion, we treated the cases where a unique time step was used. In many ocean models, however, an explicit scheme is employed for the external mode with a time step much smaller than that used for the internal mode (e.g., Blumberg and Mellor 1987; Griffies et al. 2001). In that case, it remains straightforward to ensure tracer conservation, but doing so consistently requires the continuity and free-surface elevation equations to be compatible, which is more complicated to achieve. Ensuring compatibility between these equations in order to retrieve the surface kinematic boundary condition will require incorporating a proper time averaging of the external mode depth-averaged velocity. This, as well as the use of other time-stepping algorithms, will be the subject of a future paper. Inspiration will be drawn from similar studies by Hallberg (1997) and Griffies et al. (2001). In this respect, it is worth mentioning that the lack of conservation and/or consistency associated with the use of a leapfrog algorithm through time filtering, as discussed by Griffies (2004), potentially affects our numerical scheme as well.

\section{Illustrative experiments}

A few numerical experiments using SLIM (information available online at http://www.climate.be/SLIM) are now presented. In its current configuration, the model conserves the volume and any tracer globally up to machine precision in a consistent way. The Goldsbrough-Stommel circulation, induced by freshwater fluxes, is addressed as a first illustrative experiment. We then investigate the implications of a consistency breakdown.

\section{a. Model description}

The model SLIM is described in detail by White (2007). We now briefly recall its most important features. The dynamics is split into a two-dimensional depth-averaged system for the evolution of the fastpropagating surface waves and a three-dimensional system for the vertical structure of the velocity. The same time step is used for both systems and all terms governing the propagation of inertia-gravity waves are semi-implicit in time. The elements used to interpolate the elevation and the velocity are depicted in Fig. 3. The mixed formulation $P_{1}^{\mathrm{NC}}-P_{1}$ is used for the horizontal velocity and the elevation, respectively, as described by Hanert et al. (2005). To be consistent with this choice, a linear continuous interpolation is used for the vertical velocity and all tracers. All velocity components and tracers are interpolated with linear discontinuous basis functions in the vertical.

\section{b. The Goldsbrough-Stommel circulation}

The Goldsbrough-Stommel circulation discussed by Huang and Schmitt (1993) and Huang (1993) arises from freshwater forcing and is absent in rigid-lid models. A decent rendition of the barotropic flow induced by freshwater forcing alone in the North Atlantic basin is obtained by assuming the following simple linear profile for the freshwater flux $q_{w}$ [see (3)]:

$$
q_{w}=-q_{w 0}\left[1-2 \frac{\left(y-y_{s}\right)}{\left(y_{n}-y_{s}\right)}\right]
$$

where $y$ is the meridional coordinate and $y_{s}$ and $y_{n}$ are the southern and northern coordinates of the basin, respectively. The freshwater flux magnitude is given by $q_{w 0}$. This linear profile is an idealization of observations (Huang and Schmitt 1993) and integrates to zero over the domain so that the total volume remains constant. We aim at comparing our results with those previously 


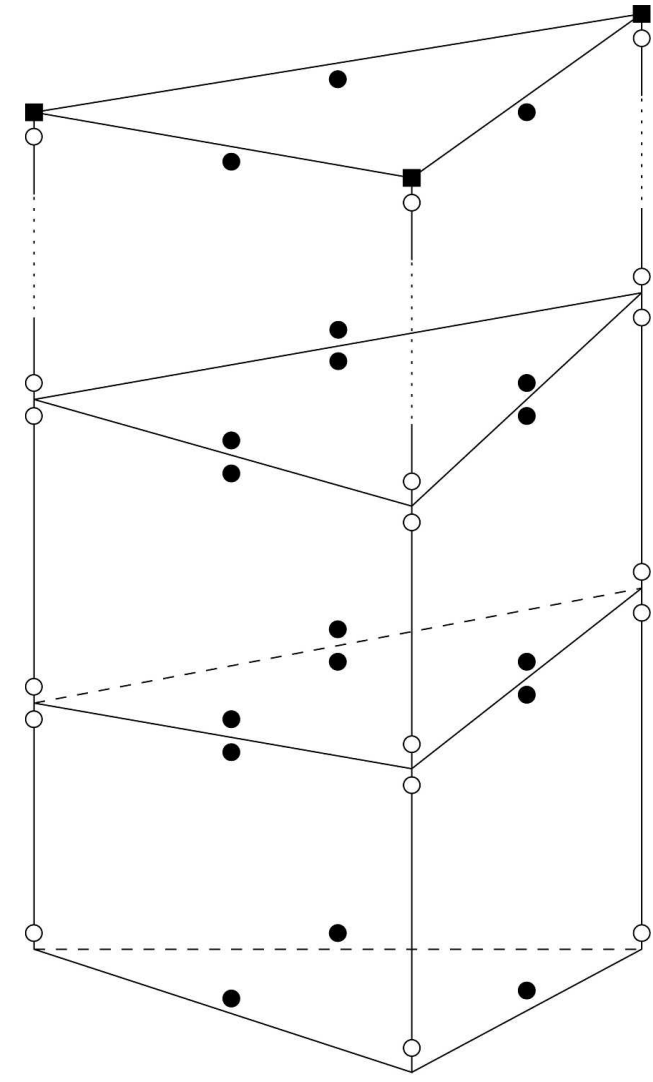

- $(u, v)$

$\circ w$ and $C$

- $\eta$

FIG. 3. Locations of nodes within a column split into prisms. The top triangle is the surface triangle. The free-surface elevation $\eta$ is linear and continuous. The horizontal velocity is linear nonconforming in the horizontal and linear discontinuous in the vertical (indicated by two nodes sharing a common physical edge). The discontinuous representation in the vertical is particularly well suited for shear regions, as it commonly occurs in baroclinic flows. The vertical velocity and tracers are linear everywhere, yet discontinuous in the vertical.

presented by Huang (1993) and Griffies et al. (2001). In both of those studies, the models were set up in spherical coordinates on a basin confined between the equator and $60^{\circ} \mathrm{N}$ and extending $60^{\circ}$ zonally. Our model was run on a square basin of size $5000 \mathrm{~km}$ with a constant depth of $4000 \mathrm{~m}$. Following Huang (1993), the coefficients of horizontal and vertical viscosity are $5 \times 10^{4}$ and $10^{-4} \mathrm{~m}^{2} \mathrm{~s}^{-1}$, respectively. The equations are solved in Cartesian coordinates on a $\beta$ plane centered at $30^{\circ} \mathrm{N}$. Neglecting the earth curvature on these scales is questionable. However, the objective of this experiment is twofold. First, we want to assess the model's ability to naturally handle freshwater fluxes as a simple forcing term in the elevation equation. Second, we want to evaluate the model's response in terms of surface salinity due to a local volume variation.

As shown in Fig. 4, our results compare well to those of Huang (1993) and Griffies et al. (2001). Three meshes were used; each one with increased resolution along the western boundary (see Fig. 4's caption for the details on the mesh resolution). All meshes comprise four evenly spaced layers of prisms. Since the resulting flow is barotropic, the number of layers has no effect on the numerical result. Both the anticyclonic subpolar and cyclonic subtropical gyres are well represented. The separation between both gyres lies at three fifths of the domain extent, in good agreement with the results of Huang (1993) and Griffies et al. (2001). The barotropic volume transport is less than $1 \mathrm{~Sv}\left(1 \mathrm{~Sv}=10^{6}\right.$ $\mathrm{m}^{3} \mathrm{~s}^{-1}$ ), which is only a few percent of the wind-driven and thermally driven transport. However, as hinted at by Fig. 5 representing the surface salinity after $3 \mathrm{yr}$, this tiny freshwater flux can potentially drive a strong threedimensional baroclinic circulation. The freshwater input and output locally cause an increase and a decrease, respectively, in the ocean volume. This, in turn, locally dilutes and concentrates the salt and a horizontal salinity gradient builds up at the surface. This dilutionconcentration effect is only due to a variation in the volume of the ocean. The tracer flux at the sea surface is zero and the total salt contents is conserved. In our experiment, salinity acts as a passive tracer: it does not feed back the flow. The coefficients of the horizontal and vertical eddy diffusivities are $10^{3}$ and $10^{-4} \mathrm{~m}^{2} \mathrm{~s}^{-1}$, respectively. For thorough studies of the freshwaterdriven baroclinic circulation, see Huang (1993), Huang and Chou (1994), and Rahmstorf (1996).

\section{c. When consistency breaks down}

The results shown thus far have been obtained by solving the consistent discrete equations, with nodes locations depicted in Fig. 3. We now study cases in which the elements for the vertical velocity $w$ and the tracer $C$ are different and whereby consistency breaks down. In all experiments described in this section, the elements used for the vertical velocity and the elevation are those depicted in Fig. 3. We use different elements for the tracer.

In Fig. 6, three situations are compared. The model is run on a $10-\mathrm{km}$-wide and 20-m-deep square basin. The initial elevation field is a 2-m-high Gaussian that we let evolve freely as a gravity wave over 1000 time steps of $72 \mathrm{~s}$. The initial tracer concentration is 1 and should remain equal to 1 at all time (there is no boundary flux and no source term). The surface integral in (28) is 
FEBRUARY 2008

WHITE ET AL.

435
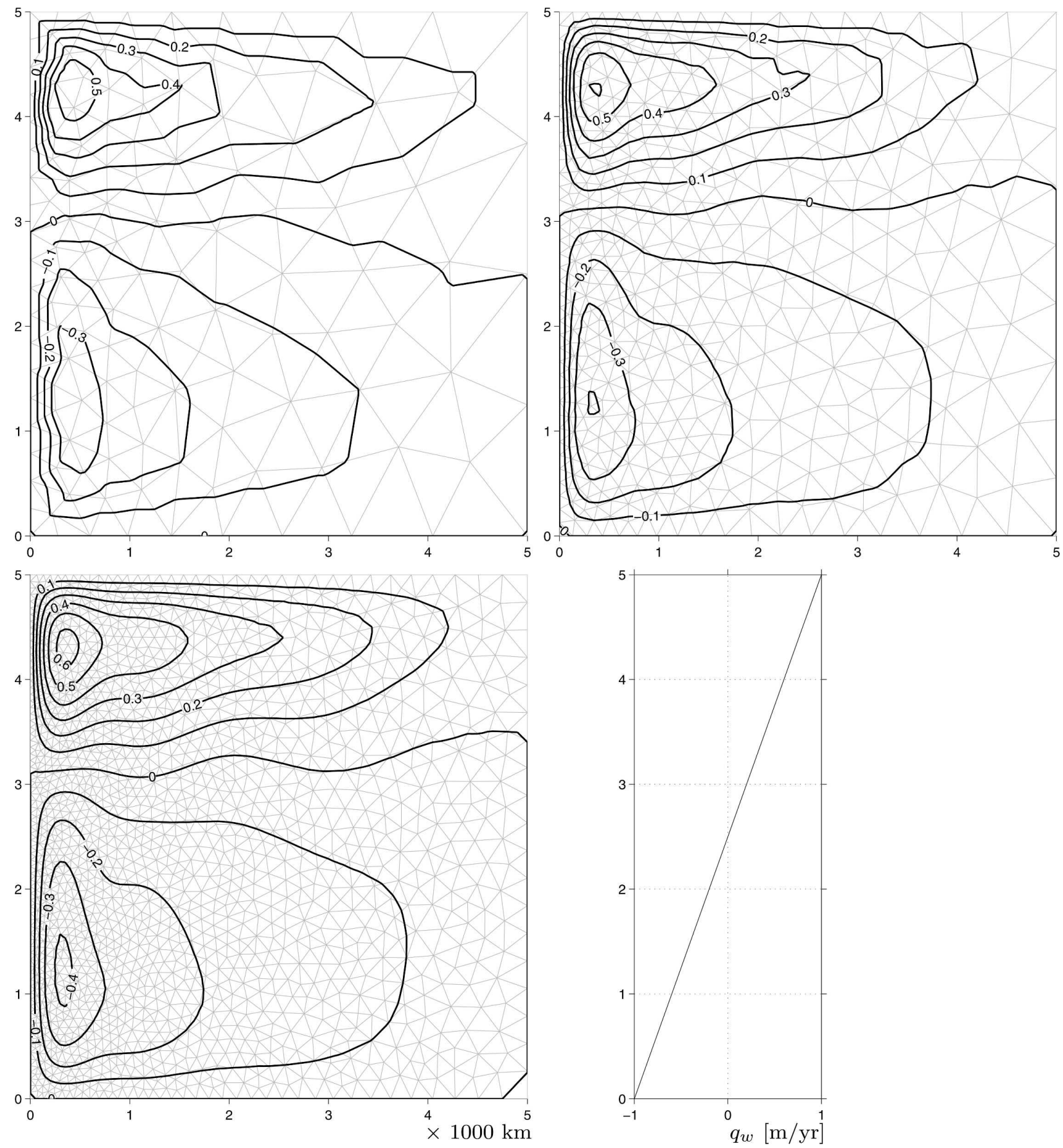

FIG. 4. Barotropic streamfunction (Xv; $1 \mathrm{~Sv}=10^{6} \mathrm{~m}^{3} \mathrm{~s}^{-1}$ ) for the freshwater-induced Goldsbrough-Stommel circulation on meshes with decreasing element sizes. The freshwater forcing $q_{w}$ varies linearly in $y$ between -1 and $1 \mathrm{~m} \mathrm{yr}^{-1}$, respectively corresponding to evaporation in the south and precipitation in the north. Numerical experiments are carried out in a square basin of 5000-km size and $4000-\mathrm{m}$ depth. Equations are solved on a $\beta$ plane centered at $30^{\circ} \mathrm{N}$. The coefficients of horizontal and vertical eddy viscosity are $5 \times$ $10^{4}$ and $10^{-4} \mathrm{~m}^{2} \mathrm{~s}^{-1}$, respectively. Meshes are refined along the western boundary of the basin. Resolution varies between 250 and 1000 $\mathrm{km}$ for the coarse mesh, between 125 and $500 \mathrm{~km}$ for the intermediate mesh, and between 62.5 and $250 \mathrm{~km}$ for the fine mesh. The coarse mesh contains 215 elements. Upon refinement, the number of elements roughly quadruples. Five layers are used in the vertical. 

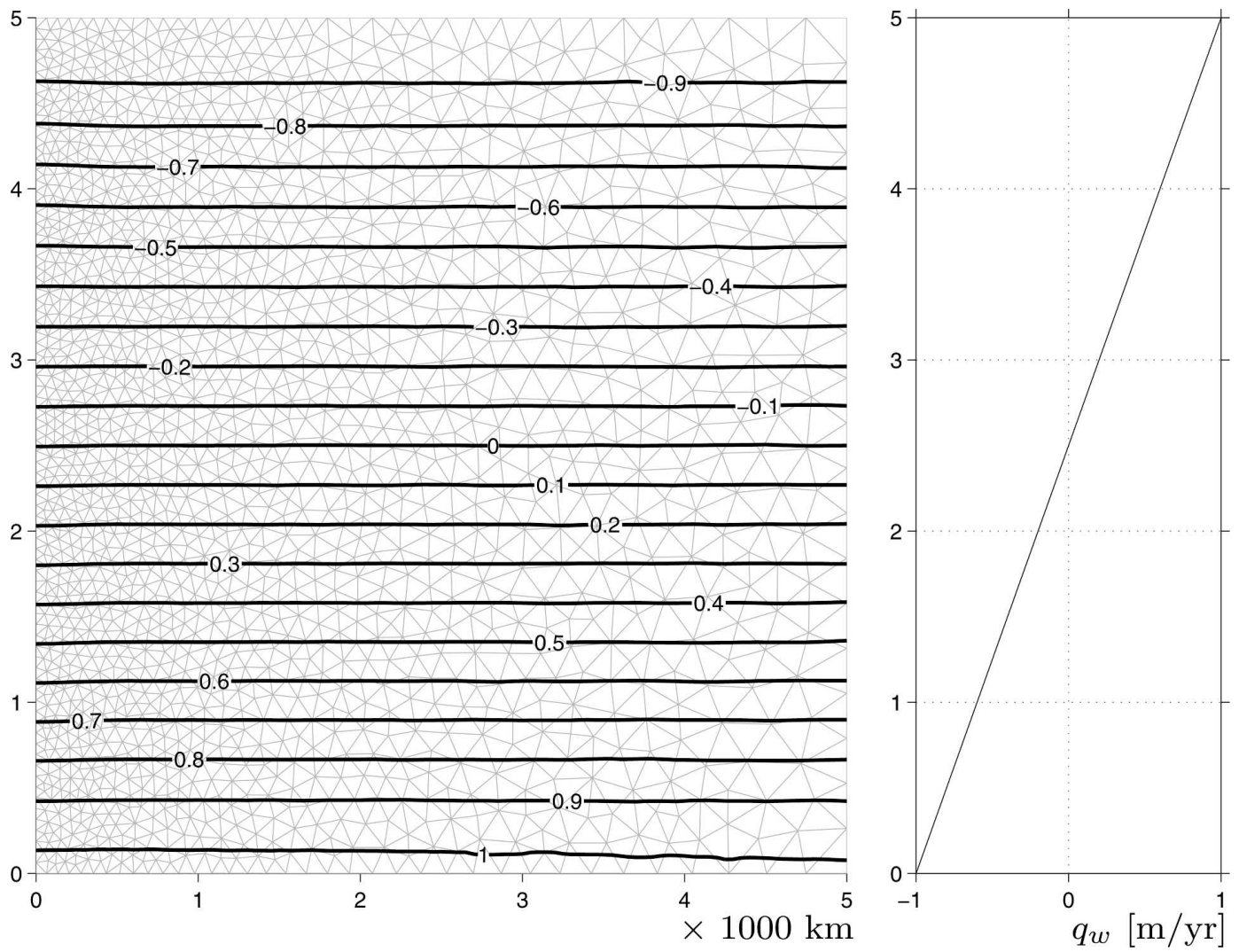

FIG. 5. Deviation of surface salinity (psu) from the reference value after a 3-yr run. In this experiment, salinity acts as a passive tracer: it does not feed back the flow. The salinity gradient buildup has the potential of driving a strong baroclinic circulation. Five layers are used in the vertical.

discarded to ensure global tracer conservation in all situations. All simulations are carried out on the threedimensional mesh shown in Fig. 7, comprising five evenly spaced layers of prisms. In the first experiment, we use the same element for $w$ and $C$. All consistency conditions are fulfilled and this is verified numerically. The deviations in the surface tracer concentration are zero (up to machine precision). In the second and third experiments, the element for $C$ is linear nonconforming and remains linear discontinuous in the vertical. The nonconforming representation in the horizontal is particularly well suited for advection-dominated flows (Hanert et al. 2004), which is the reason behind this choice. When solving the advection equation for the tracer (i.e., without diffusion), the deviations at the surface reach very high values that are unbounded numerically and grow unstably (see the second experiment in Fig. 6). In the third experiment, horizontal diffusion is added $\left(\kappa=10 \mathrm{~m}^{2} \mathrm{~s}^{-1}\right)$, which drastically reduces the deviations at the surface. Since the largest deviations are typically confined within the upper layers of the domain, they could be alleviated by choosing an appropriate diffusion coefficient.
In Fig. 8, similar experiments (without diffusion) are carried out on domains with spatial scales ranging from $10 \mathrm{~km}$ (shelf scale) to $1000 \mathrm{~km}$ (basin scale) with increasing depths, yet setting off all experiments with the same 2-m-high Gaussian (the parameters of the simulation are recalled in the figure). These runs were carried out in an attempt to gain some insight into the effects of a consistency breakdown on flows spanning a wide range of spatial scales. As we have already seen, the consequences are quite dramatic for the smallest domain (which is the same experiment as that corresponding to the middle panel in Fig. 6). For larger domains, the surface deviations remain below $1 \%$. Since the deviations of the tracer are caused by the inconsistent treatment of the advection terms, it might be expected that as soon as those terms grow larger in magnitude, those deviations will increase. For the small domain, the flow speed is on the order of $0.3 \mathrm{~m} \mathrm{~s}^{-1}$ while flow speeds decrease to roughly 0.08 and $0.01 \mathrm{~m} \mathrm{~s}^{-1}$ for the intermediate and larger domains, respectively. If we set the order of magnitude of the advection terms to 1 for the small domain, advection has relative magnitudes of $7 \times 10^{-3}$ and $10^{-5}$ for the intermediate and larger 
ELEMENTS FOR THE VERTICAL VELOCITY $w(\bullet)$ AND THE TRACER $C(\bigcirc)$
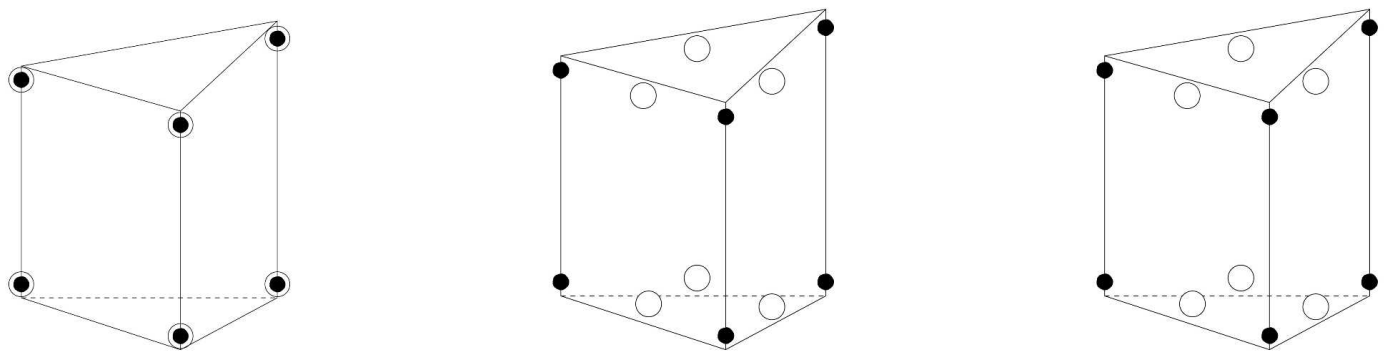

Surface tracer CONCENTRATion (DEViation From 1)

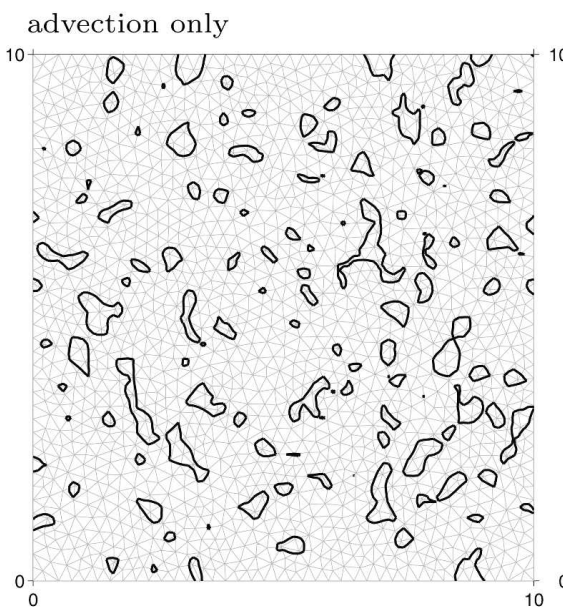
advection only advection + diff. $\left(\kappa=10 \mathrm{~m}^{2} \mathrm{~s}^{-1}\right)$

Min: $2.1 \times 10^{-14}$

Max: $9.9 \times 10^{-14}$

Contour at $5 \times 10^{-14}$ advection only

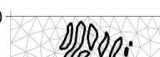

:-

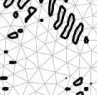

\section{D.}
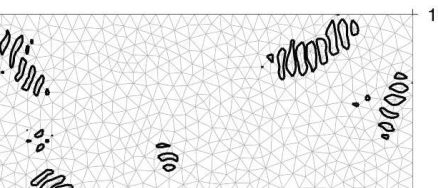

$\sqrt{10} \approx$
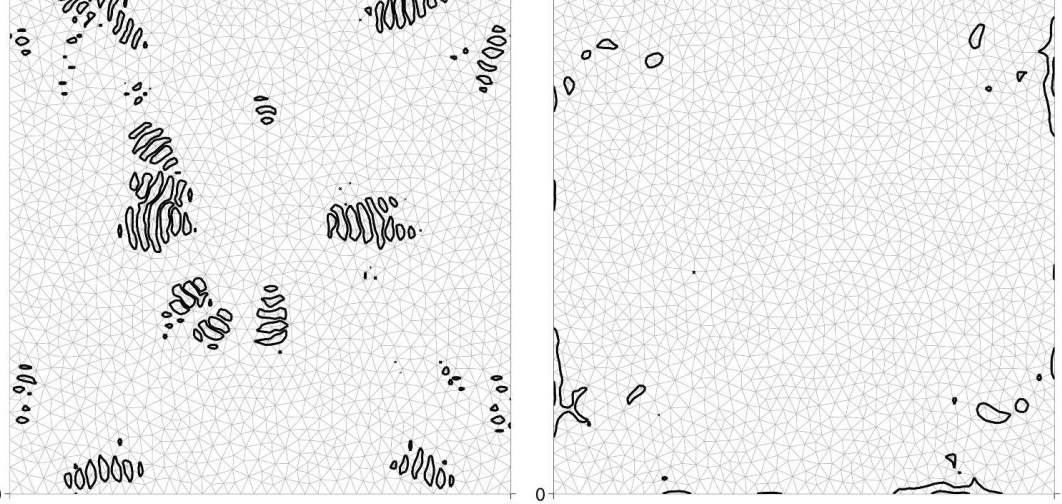

i.

\begin{abstract}
Min: $-1.8 \times 10^{2}$
\end{abstract}
Max: $1.8 \times 10^{2}$

Contour at $\pm 0.2 \times 10^{2}$

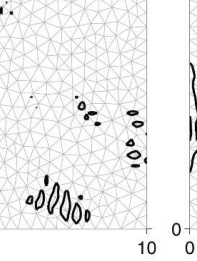

Min: $-3.7 \times 10^{-2}$

Max: $1.6 \times 10^{-2}$

Contour at $\pm 1 \times 10^{-2}$

RELATIVE DEVIATION IN TOTAL TRACER CONTAINED IN $\Omega$ OVER 1000 Time STEPS $\left(\times 10^{-13}\right)$

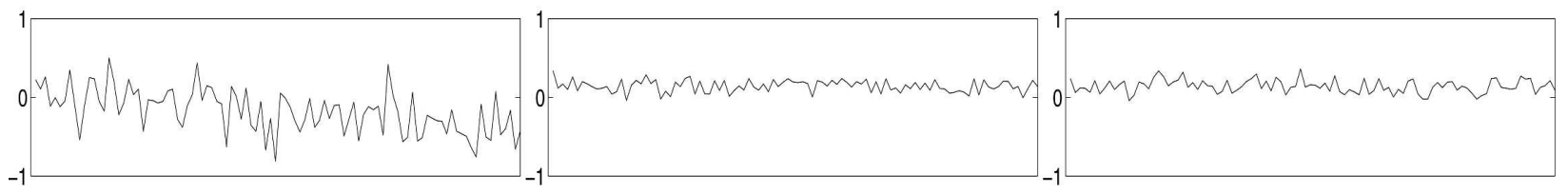

FIG. 6. Illustration of the consistency breakdown incurred when using different elements for the vertical velocity $w$ and the tracer $C$. In all simulations, the tracer concentration is initially set to 1 and should remain equal to 1 at all time. The surface flux term [see (28)] is discarded to ensure global conservation in all experiments (see bottom panels). The domain is $10 \mathrm{~km}$ wide and $20 \mathrm{~m}$ deep. The initial elevation field is a 2-m-high Gaussian. The time step is $72 \mathrm{~s}$. We use the $P_{1}$ element for the elevation in all runs. The three-dimensional mesh is described and shown in Fig. 7. We clearly see that using the same elements for $w$ and $C$ consistently ensures global conservation (surface deviations are zero in machine precision).

domains, respectively. This sheds light on the results obtained in Fig. 8.

Modeling flows in coastal and shelf regions where advection is dominant definitely requires the use of a consistent spatial scheme. Failing to do so may not only generate spurious currents but also numerical instabilities. Last, one might argue that, for the large-scale ocean circulation, inconsistency is not problematic and could be easily tackled by adding some horizontal diffusion. We do contend, however, that, even in those cases where advection is weak, conservation and consistency must be achieved to machine precision if the model is to be trusted at all for climate simulations, particularly for those models coupled with bio- 


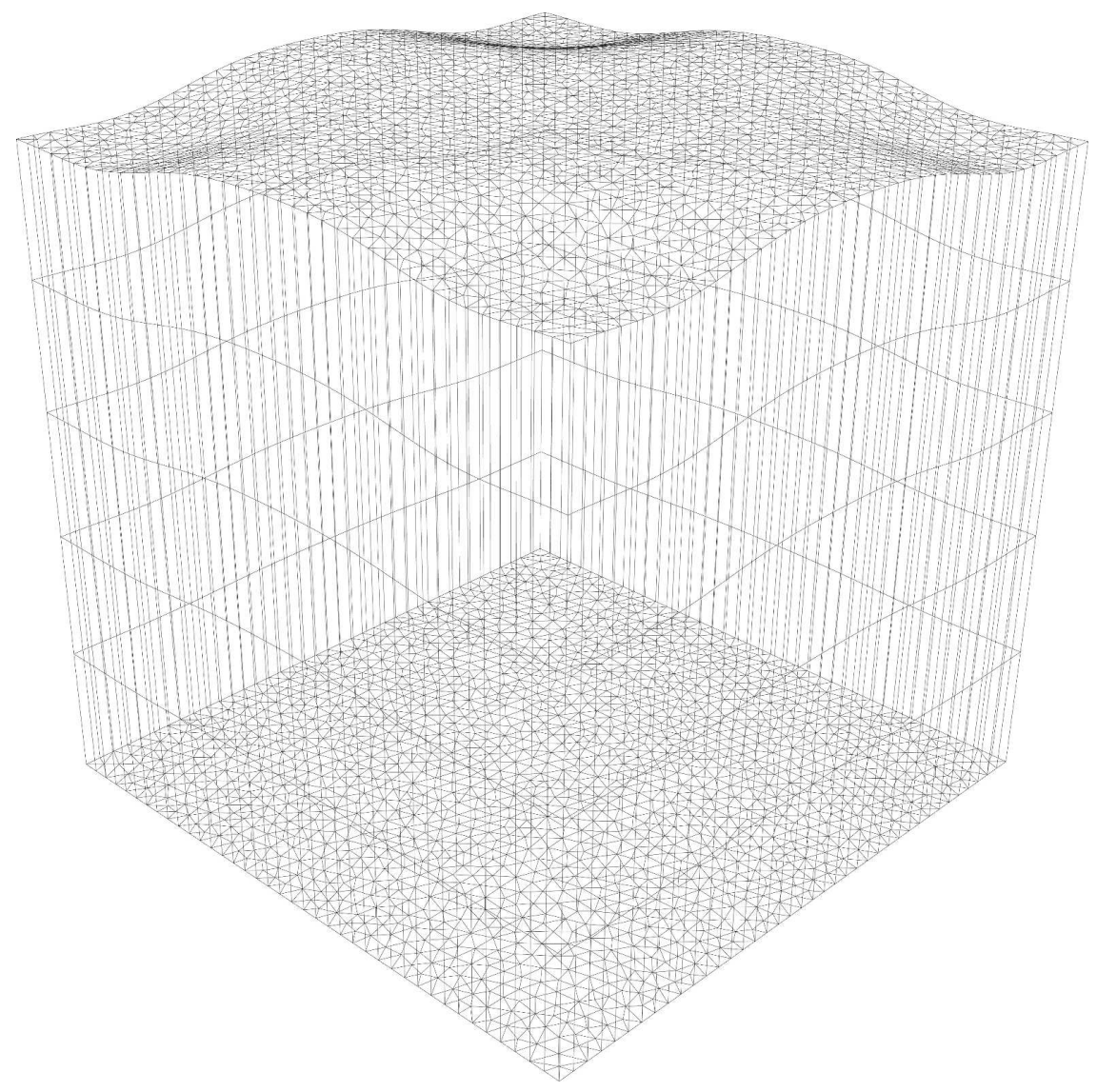

FIG. 7. Three-dimensional mesh used for the numerical experiments described in Figs. 6 and 7. The mesh has a resolution of $250 \mathrm{~m}$ in the horizontal and comprises five evenly spaced layers in the vertical. The depth-width ratio has been rescaled for clarity but the ratio of the free-surface elevation to the depth is the same as that obtained with the physical dimensions (depth of $20 \mathrm{~m}$ and free-surface undulations of the order of $1 \mathrm{~m}$ ).

geochemical cycles (Gnanadesikan 1999; Griffies et al. 2005). If these properties are not strictly satisfied, the physical integrity of the numerical solution will eventually degrade down to an unpredictable state.

\section{Conclusions}

In this paper, we have summarized sufficient conditions in terms of the finite-element discretization of the free-surface elevation, continuity, and tracer equations to consistently conserve any tracer globally. A consistent (or discretely compatible) spatial scheme is defined as one that maintains the uniform tracer concentration set initially (when there is no boundary flux and no source term). The following conditions must be fulfilled to satisfy those properties. (i) The same interpolation must be used in the horizontal for the elevation, the vertical velocity, and the tracer. (ii) The same interpolation in the vertical must be used for the vertical ve- locity and the tracer. (iii) The mesh update procedure must satisfy the discrete geometric conservation law. (iv) The mesh velocity at the surface must be equal to $\partial \eta / \partial t$. These considerations must be complemented by the necessity of choosing a stable finite-element pair for the primitive shallow-water equations.

We described a time-stepping algorithm that satisfies all compatibility constraints and, so, ensures conservation and consistency. The algorithm works in both cases of nonlinear and linearized free-surface elevation equations. When mode splitting is opted for, the horizontal velocity must be corrected prior to computing the vertical velocity in order to ensure a discrete compatibility between the elevation and continuity equations. We also suggest that there is a need for future research in order to design a consistent split-explicit algorithm.

Several numerical experiments were carried out to show the model's ability to respond to freshwater forcing. In particular, we showed how variations in the do- 


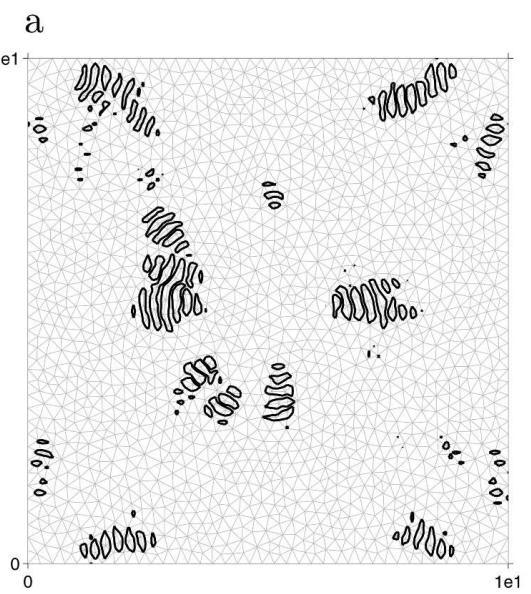

$10 \mathrm{~km} \times 10 \mathrm{~km}$

$20 \mathrm{~m}$ deep

$\mathcal{O}(\eta / d)=10^{-1}$

1000 time steps $(\Delta t=72 \mathrm{~s})$ b

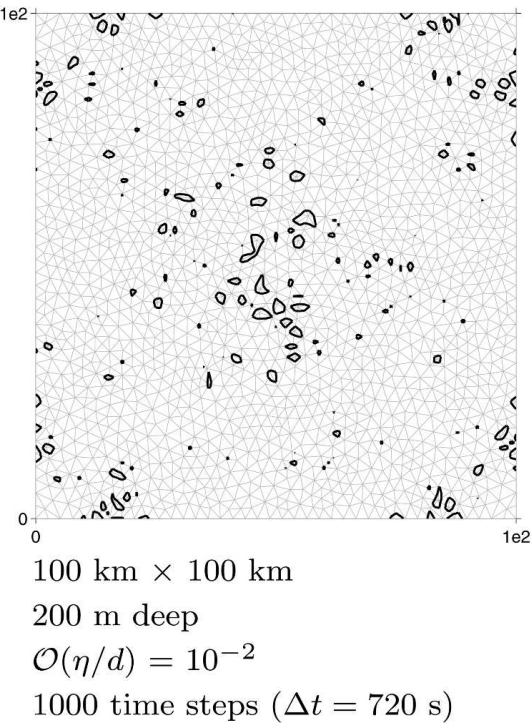

c

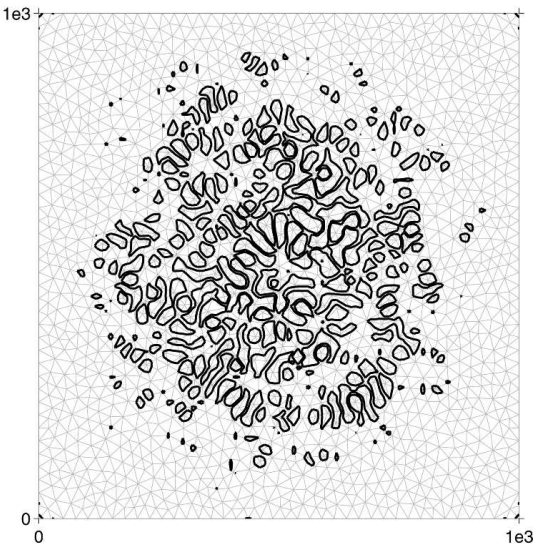

$1000 \mathrm{~km} \times 1000 \mathrm{~km}$

$2000 \mathrm{~m}$ deep

$\mathcal{O}(\eta / d)=10^{-3}$

1000 time steps $(\Delta t=7200 \mathrm{~s})$

FIG. 8. The deviation of the tracer concentration at the surface of the domain. The tracer is initially set to 1 throughout the domain and should remain equal to 1 at all time. In all runs, we used the mesh shown in Fig. 7, with appropriate scaling: (a) shelf scale, contours are drawn for deviations of -20 and 20 (resolution is $250 \mathrm{~m}$ ); (b) intermediate scale, contours are drawn for deviations of -0.005 and 0.005 (resolution is $2.5 \mathrm{~km}$ ); and (c) basin scale, contours are drawn for deviations of -0.001 and 0.001 (resolution is $25 \mathrm{~km}$ ). The corresponding simulation features are given below each panel. The initial elevation field is a 2-m-high Gaussian.

main volume naturally lead to dilution and concentration of salt, while conserving its total content. We then performed a series of experiments in which consistency between the vertical velocity and the concentration was deliberately broken down. We conclude by recommending the use of a consistent scheme for all flow regimes.

Acknowledgments. Laurent White and Eric Deleersnijder are a research fellow and a research associate, respectively, with the Belgian National Fund for Scientific Research (FNRS). The present study was carried out within the scope of the project A SecondGeneration Model of the Ocean System, which is funded by the Communauté Française de Belgique, as Actions de Recherche Concertées, under Contract ARC 04/09-316. This work is a contribution to the construction of SLIM (information online at http://www. climate.be/SLIM).

\section{APPENDIX}

\section{Downward Integration of the Continuity Equation}

In the body of the article, the continuity equation is integrated upward after imposing the kinematic boundary condition at the seabed. By doing so, the discrete surface kinematic boundary condition is automatically retrieved. Herein, we show that an equivalent result is obtained by integrating the continuity equation downward after imposing the kinematic boundary condition at the surface. Let us consider the discrete variational statement for the continuity equation, inferred from (25), in which boundary conditions are yet to be prescribed. We have

$$
\begin{aligned}
& -\int_{\Omega^{h}}\left(\mathbf{u}^{h} \cdot \nabla \psi_{i}^{w}+w^{h} \frac{\partial \psi_{i}^{w}}{\partial z}\right) d \Omega \\
& +\int_{\Gamma_{b}^{h}} \psi_{i}^{w}\left(\mathbf{u}^{h} \cdot \mathbf{n}+w^{h} n_{z}\right) \mathrm{d} \tau \\
& +\int_{\Gamma_{s}^{h}} \psi_{i}^{w}\left(\mathbf{u}^{h} \cdot \mathbf{n}+w^{h} n_{z}\right) d \tau=0, \quad \forall i=1,2, \ldots, N_{w} .
\end{aligned}
$$

Imposing the discrete counterpart of the surface kinematic boundary condition in (7) gives rise to

$$
\begin{aligned}
& -\int_{\Omega^{h}}\left(\mathbf{u}^{h} \cdot \nabla \psi_{i}^{w}+w^{h} \frac{\partial \psi_{i}^{w}}{\partial z}\right) d \Omega \\
& +\int_{\Gamma_{b}^{h}} \psi_{i}^{w}\left(\mathbf{u}^{h} \cdot \mathbf{n}+w^{h} n_{z}\right) d \tau \\
& +\int_{\Gamma_{s}^{h}} \psi_{i}^{w} \frac{\partial \eta^{h}}{\partial t} n_{z} d \tau=0, \quad \forall i=1,2, \ldots, N_{w} .
\end{aligned}
$$


We may now add up all components of the above expression pertaining to those test functions $\psi_{i}^{w}$ sharing the same two-dimensional support (the indices belong to the set $I$ ). This yields the following expression, written for the two-dimensional projection of $\psi_{i}^{w}(i \in I)$, noted $\psi_{I}^{\omega, 2 D}$ :

$$
\begin{aligned}
-\int_{\Omega^{h}} \mathbf{u}^{h} \cdot \nabla \psi_{I}^{w, 2 D} d \Omega & +\int_{\Gamma_{b}^{h}} \psi_{I}^{w, 2 D}\left(\mathbf{u}^{h} \cdot \mathbf{n}+w^{h} n_{z}\right) d \tau \\
& +\int_{\Gamma_{s}^{h}} \psi_{I}^{w, 2 D} \frac{\partial \eta^{h}}{\partial t} n_{z} d \tau=0 .
\end{aligned}
$$

If we choose the basis functions for the vertical velocity such that $\psi_{I}^{w, 2 D}=\phi_{i}$, where $\phi_{i}$ is the two-dimensional elevation basis function associated with the same nodal position in the $(x, y)$ plane, the first and third terms of the above equation are identically equal to the discrete elevation equation [(24)], which leaves us with

$$
\int_{\Gamma_{b}^{h}} \psi_{I}^{w, 2 D}\left(\mathbf{u}^{h} \cdot \mathbf{n}+w^{h} n_{z}\right) d \tau=0
$$

namely, the discrete counterpart of the kinematic boundary condition at the seabed [(6)].

\section{REFERENCES}

Aizinger, V., and C. Dawson, 2002: A discontinuous Galerkin method for two-dimensional flow and transport in shallow water. Adv. Water Resour., 25, 67-84.

Berger, R. C., and S. E. Howington, 2002: Discrete fluxes and mass balance in finite elements. J. Hydraul. Eng., 128, 87-92.

Bernard, P.-E., N. Chevaugeon, V. Legat, E. Deleersnijder, and J.-F. Remacle, 2007: High-order $h$-adaptive discontinuous Galerkin methods for ocean modeling. Ocean Dyn., 57, 109 121.

Blumberg, A. F., and G. L. Mellor, 1987: A description of a threedimensional coastal ocean circulation model. ThreeDimensional Coastal Ocean Models, N. S. Heaps, Ed., Coastal and Estuarine Series, Vol. 4, Amer. Geophys. Union, $1-16$.

Campin, J.-M., A. Adcroft, C. Hill, and J. Marshall, 2004: Conservation of properties in a free-surface model. Ocean Modell., 6, 221-244.

Casulli, V., and R. A. Walters, 2000: An unstructured grid, threedimensional model based on the shallow-water equations. Int. J. Numer. Methods Fluids, 32, 331-346.

Cushman-Roisin, B., and C. E. Naimie, 2002: A 3D finite-element model of the Adriatic tides. J. Mar. Syst., 37, 279-297.

Danilov, S., G. Kivman, and J. Schröter, 2004: A finite-element ocean model: Principles and evaluation. Ocean Modell., 6, $125-150$.

,-- , and J. Schröter, 2005: Evaluation of an eddy-permitting finite-element ocean model in the North Atlantic. Ocean Modell., 10, 35-49.

Dawson, C., J. J. Westerink, J. C. Feyen, and D. Pothina, 2006: Continuous, discontinuous and coupled discontinuouscontinuous Galerkin finite element methods for the shallow water equations. Int. J. Numer. Methods Fluids, 52, 63-88.
Deleersnijder, E., 1993: Numerical mass conservation in a freesurface sigma coordinate marine model with mode splitting. J. Mar. Syst., 4, 365-370.

- , and J.-M. Campin, 1995: On the computation of the barotropic mode of a free-surface world ocean model. Ann. Geophys., 13, 675-688.

Donea, J., A. Huerta, J.-P. Ponthot, and A. Rodríguez-Ferran, 2004: Arbitrary Lagrangian-Eulerian methods. Encyclopedia of Computational Mechanics, E. Stein, R. de Borst, and T. J. R. Hughes, Eds., John Wiley and Sons, 1-25.

Farhat, C., P. Geuzaine, and C. Grandmont, 2001: The discrete geometric conservation law and the nonlinear stability of ALE schemes for the solution of flow problems on moving grids. J. Comput. Phys., 174, 669-694.

Flaherty, J. E., L. Krivodonova, J.-F. Remade, and M. S. Shephard, 2002: Aspects of discontinuous Galerkin methods for hyperbolic conservation laws. Finite Elements Anal. Design, 38, 889-908.

Ford, R., C. C. Pain, M. D. Piggott, A. J. H. Goddard, C. R. E. de Oliveira, and A. P. Umpleby, 2004a: A nonhydrostatic finiteelement model for three-dimensional stratified oceanic flows. Part I: Model formulation. Mon. Wea. Rev., 132, 2816-2831.

,,,,----- , and,$- 2004 \mathrm{~b}$ : A nonhydrostatic finite-element model for three-dimensional stratified oceanic flows. Part II: Model validation. Mon. Wea. Rev., 132, 2832 2844.

Formaggia, L., and F. Nobile, 2004: Stability analysis of secondorder time accurate schemes for ALE-FEM. Comput. Methods Appl. Mech. Eng., 193, 4097-4116.

Fortunato, A. B., A. M. Baptista, and R. A. Luettich, 1997: A three-dimensional model of tidal currents in the mouth of the Tagus estuary. Cont. Shelf Res., 17, 1689-1714.

Fringer, O. B., M. Gerritsen, and R. L. Street, 2006: An unstructured-grid, finite-volume, non-hydrostatic, parallel coastal ocean simulator. Ocean Modell., 14, 139-173.

Gnanadesikan, A., 1999: Numerical issues for coupling biological models with isopycnal mixing schemes. Ocean Modell., 1, $1-15$.

Gorman, G. J., M. D. Piggott, C. C. Pain, C. R. E. Oliveira, A. P. Umpleby, and A. J. H. Goddard, 2006: Optimisation based bathymetry approximation through constrained unstructured mesh adaptivity. Ocean Modell., 12, 436-452.

Greenberg, D. A., F. E. Werner, and D. R. Lynch, 1998: A diagnostic finite-element ocean circulation model in sphericalpolar coordinates. J. Atmos. Oceanic Technol., 15, 942-958.

Griffies, S. M., 2004: Fundamentals of Ocean Climate Models. Princeton University Press, $496 \mathrm{pp}$.

, and Coauthors, 2000: Developments in ocean climate modelling. Ocean Modell., 2, 123-192.

— R. C. Pacanowski, M. Schmidt, and V. Balah, 2001: Tracer conservation with an explicit free-surface method for $z$ coordinate ocean models. Mon. Wea. Rev., 129, 1081-1098.

_ and Coauthors, 2005: Formulation of an ocean model for global climate simulations. Ocean Sci., 1, 45-79.

Gross, E. S., L. Bonaventura, and G. Rosatti, 2002: Consistency with continuity in conservative advection schemes for freesurface models. Int. J. Numer. Methods Fluids, 38, 307-327.

Hallberg, R., 1997: Stable split time stepping scheme for largescale ocean modeling. J. Comput. Phys., 135, 54-65.

Ham, D. A., J. Pietrzak, and G. S. Stelling, 2005: A scalable unstructured grid 3-dimensional finite volume model for the shallow water equations. Ocean Modell., 10, 153-169.

Hanert, E., V. Legat, and E. Deleersnijder, 2003: A comparison of 
three finite elements to solve the linear shallow water equations. Ocean Modell., 5, 17-35.

— D. Y. Le Roux, V. Legat, and E. Deleersnijder, 2004: Advection schemes for unstructured grid ocean modelling. Ocean Modell., 7, 39-58.

,,--- , and,- 2005 : An efficient Eulerian finite element method for the shallow water equations. Ocean Modell., 10, 115-136.

Higdon, R. L., and R. A. de Szoeke, 1997: Barotropic-baroclinic time splitting for ocean circulation modeling. J. Comput. Phys., 135, 30-53.

Hua, B.-L., and F. Thomasset, 1984: A noise-free finite element scheme for the two-layer shallow water equations. Tellus, 36A, 157-165.

Huang, R. X., 1993: Real freshwater flux as a natural boundary condition for the salinity balance and thermohaline circulation forced by evaporation and precipitation. J. Phys. Oceanogr., 23, 2428-2446.

, and R. W. Schmitt, 1993: The Goldsbrough-Stommel circulation of the World Ocean. J. Phys. Oceanogr., 23, 1277-1284.

—, and R. L. Chou, 1994: Parameter sensitivity study of the saline circulation. Climate Dyn., 9, 391-409.

Hughes, T. J. R., L. Engel, L. Mazzei, and M. G. Larson, 2000: The continuous Galerkin method is locally conservative. $J$. Comput. Phys., 163, 467-488.

Iskandarani, M., D. B. Haidvogel, and J. P. Boyd, 1995: A staggered spectral element model with application to the oceanic shallow water equations. Int. J. Numer. Methods Fluids, 20, 393-414.

,-- , and J. C. Levin, 2003: A three-dimensional spectral element model for the solution of the hydrostatic primitive equations. J. Comput. Phys., 186, 397-425.

Killworth, P. D., D. Stainforth, D. J. Webb, and S. M. Paterson, 1991: The development of a free-surface Bryan-CoxSemtner ocean model. J. Phys. Oceanogr., 21, 1333-1348.

Kinnmark, I., 1986: The Shallow Water Wave Equations: Formulation, Analysis and Applications. Lecture Notes in Engineering, Vol. 15, Springer-Verlag, 187 pp.

Kolar, R. L., J. J. Westerink, M. E. Cantekin, and C. Blain, 1994: Aspects of nonlinear simulations using shallow-water models based on the wave continuity equation. Comput. Fluids, 23, 523-538.

Kubatko, E. J., J. J. Westerink, and C. Dawson, 2006a: An unstructured grid morphodynamic model with a discontinuous Galerkin method for bed evolution. Ocean Modell., 15, 7189

,-- , and $-2006 \mathrm{~b}$ : Discontinuous Galerkin methods for advection dominated problems in shallow water flow. Comput. Methods Appl. Mech. Eng., 196, 437-451.

Labeur, R. J., and J. D. Pietrzak, 2005: A fully three dimensional unstructured grid non-hydrostatic finite element coastal model. Ocean Modell., 10, 51-67.

Legrand, S., V. Legat, and E. Deleersnijder, 2000: Delaunay mesh generation for an unstructured-grid ocean general circulation model. Ocean Modell., 2, 17-28.

, E. Deleersnijder, E. Hanert, V. Legat, and E. Wolanski, 2006: High-resolution unstructured meshes for hydrodynamic models of the Great Barrier Reef, Australia. Estuarine Coastal Shelf Sci., 68, 36-46.

- - - E. Delhez, and V. Legat, 2007: Unstructured, anisotropic mesh generation for the northwestern European continental shelf, the continental slope and the neighboring ocean. Cont. Shelf Res., doi:10.1016/j.csr.2007.01.009.
Le Roux, D. Y., 2001: A new triangular finite-element with optimum constraint ratio for compressible fluids. SIAM J. Sci. Comput., 23, 66-80.

- , 2005: Dispersion relation analysis of the $P_{1}^{\mathrm{NC}}-P_{1}$ finiteelement pair in shallow-water models. SIAM J. Sci. Comput., 27, 394-414.

- , A. Staniforth, and C. A. Lin, 1998: Finite elements for shallow-water equation ocean models. Mon. Wea. Rev., 126, 1931-1951.

— A. Sène, V. Rostand, and E. Hanert, 2005: On some spurious mode issues in shallow-water models using a linear algebra approach. Ocean Modell., 10, 83-94.

_ , V. Rostand, and B. Pouliot, 2007: Analysis of numerically induced oscillations in 2D finite-element shallow-water models. Part I: Inertia-gravity waves. SIAM J. Sci. Comput., 29, 331-360.

Lynch, D. R., and W. R. Gray, 1979: A wave equation model for finite element tidal computations. Comput. Fluids, 7, 207228

_ , and F. E. Werner, 1987: Three-dimensional hydrodynamics on finite elements. Part I: Linearized harmonic model. Int. J. Numer. Methods Fluids, 7, 871-909.

_ and _ 1991: Three-dimensional hydrodynamics on finite elements. Part II: Non-linear time-stepping model. Int. J. Numer. Methods Fluids, 12, 507-533.

— , and C. E. Naimie, 1993: The $\mathrm{M}_{2}$ tide and its residuals on the outer banks of the Gulf of Maine. J. Phys. Oceanogr., 23, 2222-2253.

— J. T. C. Ip, C. E. Naimie, and F. E. Werner, 1996: Comprehensive coastal circulation model with application to the Gulf of Maine. Cont. Shelf Res., 16, 875-906.

Massey, T. C., and C. A. Blain, 2006: In search of a consistent and conservative mass flux for the GWCE. Comput. Methods Appl. Mech. Eng., 195, 571-587.

Miglio, E., A. Quarteroni, and F. Saleri, 1998: Finite element approximation of quasi-3D shallow water equations. Comput. Methods Appl. Mech. Eng., 174, 355-369.

Muccino, J. C., W. G. Gray, and M. G. G. Foreman, 1997: Calculation of vertical velocity in three-dimensional, shallow-water equation, finite element models. Int. J. Numer. Methods Fluids, 25, 779-802.

Myers, P. G., and A. J. Weaver, 1995: A diagnostic barotropic finite-element ocean circulation model. J. Atmos. Ocean. Technol., 12, 511-526.

Nair, R. D., S. J. Thomas, and R. D. Loft, 2005: A discontinuous Galerkin global shallow water model. Mon. Wea. Rev., 133, 876-888.

Nechaev, D., J. Schröter, and M. Yaremchuk, 2003: A diagnostic stabilized finite-element ocean circulation model. Ocean Modell., 5, 37-63.

Pain, C. C., and Coauthors, 2005: Three-dimensional unstructured mesh ocean modelling. Ocean Modell., 10, 5-33.

Piggott, M. D., C. C. Pain, G. J. Gorman, P. W. Power, and A. J. H. Goddard, 2005: $h, r$, and $h r$ adaptivity with applications in numerical ocean modelling. Ocean Modell., 10, 95-113.

Power, P. W., M. D. Piggott, F. Fang, G. J. Gorman, C. C. Pain, D. P. Marshall, A. J. H. Goddard, and I. M. Navon, 2006: Adjoint goal-based error norms for adaptive mesh ocean modelling. Ocean Modell., 15, 3-38.

Rahmstorf, S., 1996: On the freshwater forcing and transport of the Atlantic thermohaline circulation. Climate Dyn., 12, 799 811. 
Roullet, G., and G. Madec, 2000: Salt conservation, free surface and varying levels: A new formulation for ocean general circulation models. J. Geophys. Res., 105, 23 927-23 942.

Schwanenberg, D., and M. Harms, 2004: Discontinuous Galerkin finite-element method for transcritical two-dimensional shallow water flows. J. Hydraul. Eng., 130, 412-421.

_, R. Kiem, and J. Kongeter, 2000: A discontinuous Galerkin method for the shallow-water equations with source terms. Discontinuous Galerkin Methods: Theory, Computations and Applications, B. Cockburn, G. E. Karniadaki, and C.-W. Chu, Eds., Lecture Notes in Computational Science and Engineering, Vol. 11, Springer, 419-424.

Simons, T. J., 1974: Verification of numerical models of Lake Ontario. Part I: Circulation in spring and early summer. J. Phys. Oceanogr., 4, 507-523.

Walters, R. A., 1992: A three-dimensional, finite element model for coastal and estuarine circulation. Cont. Shelf Res., 12, 83-102.

, 2005: Coastal ocean models: Two useful finite element methods. Cont. Shelf Res., 25, 775-793.
—, 2006: Design considerations for a finite element coastal ocean model. Ocean Modell., 15, 90-100.

— , and F. E. Werner, 1989: A comparison of two finite element models of tidal hydrodynamics using the North Sea data set. Adv. Water Resour., 12, 184-193.

White, L., 2007: Accuracy and consistency in finite element ocean modeling. Ph.D. thesis, Université Catholique de Louvain, 164 pp. [Available online at http://edoc.bib.ucl.ac.be:81/ETDdb/collection/available/BelnUcetd-03192007-143839.]

_ , and E. Deleersnijder, 2007: Diagnoses of vertical transport in a three-dimensional finite-element model of the tidal circulation around an island. Estuarine Coastal Shelf Sci., 74, 655-669.

— J.-M. Beckers, E. Deleersnijder, and V. Legat, 2006a: Comparison between free-surface and rigid-lid finite element models of barotropic instabilities. Ocean Dyn., 56, 86-103.

— , V. Legat, E. Deleersnijder, and D. Le Roux, 2006b: A onedimensional benchmark for the propagation of Poincaré waves. Ocean Modell., 15, 101-123. 